https://doi.org/10.23913/ride.v10i19.494

Artículos Científicos

\title{
Herramientas tecnológicas en el proceso de enseñanza- aprendizaje en estudiantes de educación superior
}

Technological Tools in the Teaching-Learning Process in Higher Education

Students

Ferramentas tecnológicas no processo de ensino-aprendizagem em estudantes do ensino superior

María del Carmen Molinero Bárcenas

Universidad Autónoma de Querétaro, México carmen95@uaq.mx https://orcid.org/0000-0001-5915-9225

Ubaldo Chávez Morales Universidad Autónoma de Querétaro, México ubald.chavez@gmail.com https://orcid.org/0000-0001-5753-8934

* Autor de correspondencia: María del Carmen Molinero Bárcenas 


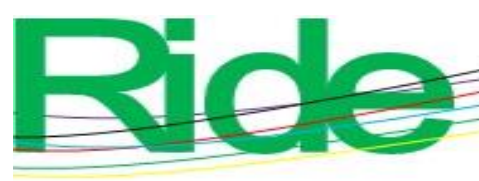

Revista Iberoamericana para la

Investigación y el Desarrollo Educativo ISSN $2007-7467$

\section{Resumen}

El objetivo de este trabajo fue investigar cuáles son las herramientas tecnológicas más utilizadas por estudiantes universitarios en una institución de educación superior e identificar cómo éstas influyen en su proceso educativo. La metodología fue cuantitativa. El instrumento utilizado fue una encuesta electrónica para recabar la información. Participaron 224 estudiantes de licenciatura y posgrado. Los datos aquí presentados tienen relación directa con el uso de las herramientas tanto a nivel escolar como individual.

Dentro de los resultados obtenidos, sobresale el uso de Microsoft Word como procesador de textos y Microsoft PowerPoint para efectuar presentaciones. En cuanto al uso de videoconferencias, son muy pocos los profesores y estudiantes que utilizan este medio. El software de comunicación que más sobresale es Skype. Por otra parte, el dispositivo que más utilizan los estudiantes para sus trabajos escolares es la computadora, pero en su vida cotidiana recurren sobre todo al smartphone. También se pudo detectar que los estudiantes utilizan cada vez más herramientas tecnológicas en la escuela y en su vida común y que estas no necesariamente concuerdan con las que el profesor recomienda y revisa en clase. Ellos mismos se dan a la tarea de buscar aplicaciones que les sirvan para sus actividades escolares. Esto se demostró con las plataformas educativas que utilizan, pues no solo han utilizado la institucional, sino que sobresalen otras como Canvas y Socrative.

En esta investigación se pudo observar que, a pesar de que existen diversas aplicaciones para presentaciones, los estudiantes siguen prefiriendo los programas de Microsoft Office para sus trabajos escolares. Esta información es relevante para los docentes, y puede ser útil para generar y aplicar estrategias diversas en el salón de clase, ya que para los alumnos estas herramientas son necesarias en su proceso educativo y pueden ayudar a mejorar su desempeño académico.

Palabras clave: aprendizaje, educación superior, enseñanza, estudiantes, herramientas tecnológicas, tecnología. 


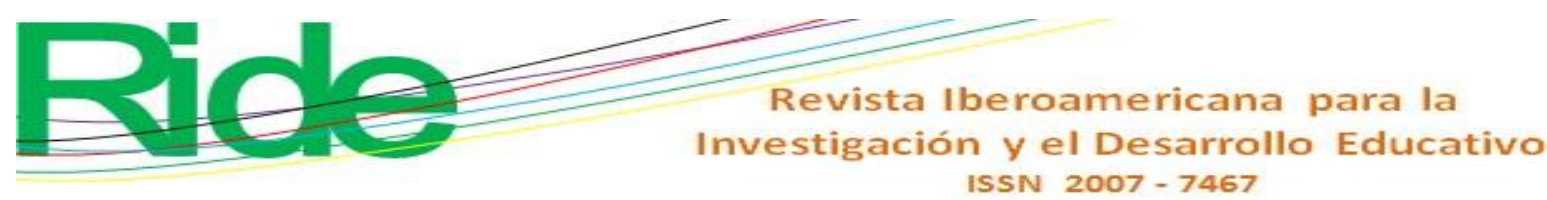

\section{Abstract}

The objective of this work was to investigate the technological tools most used by university students in a higher education institution and to identify how these influence their educational process. The methodology was quantitative. And the instrument used was an electronic survey to collect information. In total, 224 undergraduate and graduate students participated.

The results obtained show that programs such as Microsoft Word and Microsoft PowerPoint stand out. Regarding videoconferencing, there are still very few teachers and students who use it. The software that excels in this aspect is Skype. On the other hand, the device that most students use for their work at school is the computer, but the one they most use for a game or application is the telephone. Likewise, it was observed that students use more and more technological tools in the school and not only those used by teachers. They themselves are given the task of looking for applications that serve their school activities. This was demonstrated with the educational platforms that they use, since not only have they used the institutional one, but others like Canvas and Socrative stand out.

With this research it was possible to demonstrate that although there are diverse applications for presentations, students still prefer the Microsoft Office programs for their school work. This information is relevant for teachers, and can be useful to generate and apply different strategies in the classroom, since for students these tools are necessary in their educational process and can help improve their performance.

Keywords: learning, higher education, teaching, students, technological tools, technology.

\section{Resumo}

O objetivo deste trabalho foi investigar quais são os instrumentos tecnológicos mais utilizados por estudantes universitários de uma instituição de ensino superior e identificar como estes influenciam seu processo educacional. A metodologia foi quantitativa. $\mathrm{O}$ instrumento utilizado foi uma pesquisa eletrônica para coletar informações. 224 estudantes de graduação e pós-graduação participaram. Os dados aqui apresentados estão diretamente relacionados ao uso de ferramentas tanto na escola quanto no nível individual. 


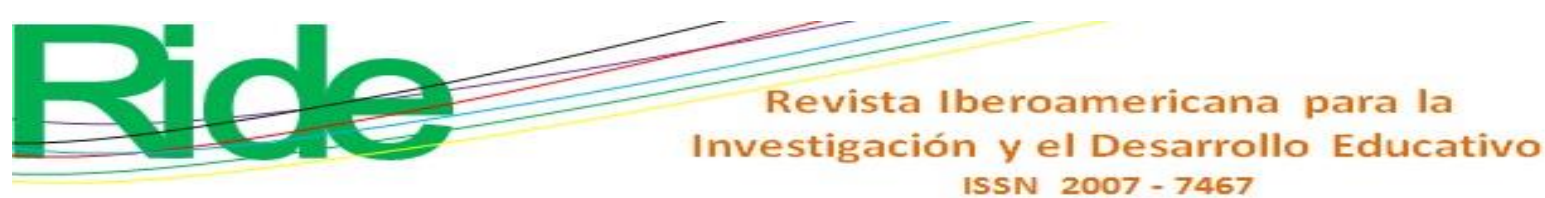

Entre os resultados obtidos, destaca-se o uso do Microsoft Word como processador de texto e Microsoft PowerPoint para fazer apresentações. Em relação ao uso de videoconferências, existem muito poucos professores e alunos que utilizam esse meio. O software de comunicação mais notável é o Skype. Por outro lado, o dispositivo que a maioria dos alunos usa para o trabalho escolar é o computador, mas em suas vidas diárias eles usam principalmente o smartphone. Foi também detectado que os alunos utilizam cada vez mais ferramentas tecnológicas na escola e na sua vida em comum e que estes não necessariamente concordam são aqueles que o professor recomenda e revê em aula. Eles mesmos recebem a tarefa de procurar aplicativos que atendam às suas atividades escolares. Isso foi demonstrado pelas plataformas educacionais que utilizam, já que não só usaram a institucional, como destacam outras como Canvas e Socrative.

Nesta pesquisa, observou-se que, embora existam várias aplicações para apresentações, os alunos ainda preferem programas do Microsoft Office para o trabalho escolar. Essa informação é relevante para os professores e pode ser útil para gerar e aplicar diferentes estratégias em sala de aula, uma vez que para os alunos essas ferramentas são necessárias em seu processo educacional e podem ajudar a melhorar seu desempenho acadêmico.

Palavras-chave: aprendizagem, ensino superior, ensino, estudantes, ferramentas tecnológicas, tecnologia.

Fecha Recepción: Marzo 2019

Fecha Aceptación: Junio 2019

\section{Introducción}

En la actualidad, hablar de herramientas tecnológicas ya no es nada nuevo. El desarrollo de aplicaciones digitales ha adoptado un ritmo trepidante. En el contexto educativo, desde que apareció la era de la computación, es bastante común que los estudiantes utilicen varias de estas herramientas al momento de realizar sus tareas académicas. Sin duda dichos recursos les han facilitado su trabajo: el tiempo que utilizan ahora es menor al que utilizaban anteriormente. Antes se tenía que ir físicamente a la biblioteca, por ejemplo; ahora ya no es necesario desplazarse, pues la consulta de material informativo puede realizarse desde casa a través de una computadora portátil o de escritorio, teléfono móvil, tableta, en fin, cualquier dispositivo que esté conectado a Internet.

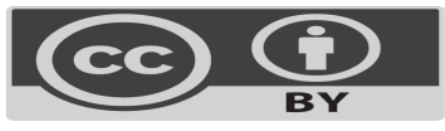



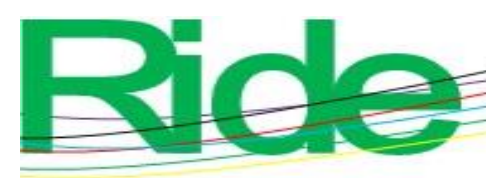

Revista Iberoamericana para la Investigación y el Desarrollo Educativo ISSN $2007-7467$

Asimismo, estas herramientas han ayudado en el proceso de enseñanza-aprendizaje, y no solo a los estudiantes, también a profesores y padres de familia. Ahora los padres se pueden involucrar más en la enseñanza de sus hijos, llegar a límites que hace algún tiempo no se podían imaginar. Cabe mencionar que en ocasiones los estudiantes manejan aplicaciones mejor que un docente, sobre todo si el docente es mayor, ya que aquellos nacieron en una época más cercana al desarrollo tecnológico imperante.

Esto ayuda a que tanto profesores como estudiantes sean capaces de aprender de una manera más dinámica, puesto que el profesor también aprende al enseñar. El nivel de competitividad será más alto a medida que se utilicen más las tecnologías de la información y comunicación (TIC) para proyectos, tareas y ejercicios en clase.

Por lo anteriormente expuesto, en este trabajo se indaga sobre las aplicaciones que utiliza un estudiante en la escuela, ya sea en la computadora portátil, el teléfono inteligente o la tableta. El objetivo es conocer dichas herramientas para darlas a conocer y que esto les pueda servir a algunos profesores al momento de elegir cuáles utilizar en el aula, y si no las conocen comenzar a familiarizarse con ellas para sus clases. Es así como las TIC han ayudado a realizar el trabajo fácil y amenamente.

\section{Objetivos}

- Investigar cuáles son las herramientas tecnológicas más utilizadas por estudiantes universitarios en una institución de educación superior.

- Identificar cómo estas herramientas influyen en su proceso educativo.

- $\quad$ Enunciar las herramientas más sobresalientes para que los docentes puedan generar y aplicar estrategias diversas en el salón de clase y con ellas ayudar a mejorar el desempeño de los estudiantes.

- $\quad$ Especificar las herramientas tecnológicas en las que el docente se debe seguir actualizando. 


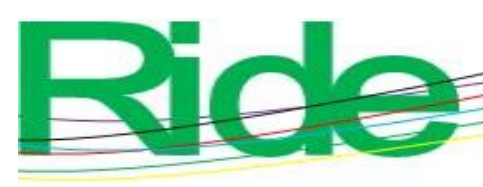

Revista Iberoamericana para la

Investigación y el Desarrollo Educativo ISSN $2007-7467$

\section{Problema}

El excesivo número de herramientas digitales que existen hoy en día hace que los estudiantes puedan utilizar algunas para la escuela y otras para el uso personal, además de que provoca que los docentes no sepan qué herramientas utilizar en clase, ya sea para trabajos, tareas o proyectos.

El diverso uso de dichas herramientas puede hacer que el desempeño del estudiante no sea suficiente a la hora de entregar trabajos, tareas o proyectos.

\section{La era de la tecnología}

En los últimos 20 años se ha ido modificando la tecnología, tanto en computadoras como en teléfonos, tabletas y demás dispositivos electrónicos que los estudiantes han utilizado y de los que, al manejarlos tan bien, se han ido apropiando.

En el año 2006 se incluyó en el currículum el tratamiento de la información y la competencia digital como aprendizaje imprescindible. Al terminar la escolarización obligatoria, los estudiantes deberán alcanzar lo siguiente:

El tratamiento de la información y la competencia digital implica ser una persona autónoma, eficaz, responsable, crítica y reflexiva al seleccionar, tratar y utilizar la información disponible, contrastándola cuando es necesario y respetar las normas de conducta acordadas socialmente para regular el uso de la información y sus fuentes en los distintos soportes (Ministerio de Educación y Ciencia [MEC], 2006).

La competencia digital en los docentes, según la Organización de las Naciones Unidas para la Educación, la Ciencia y la Cultura [Unesco] (2008), debe ser el propósito de la formación, dado que los estudiantes deben adquirir competencia digital y del tratamiento de la información explícita en el currículum. Para ello, la formación del profesorado debería dirigirse hacia un modelo que encaje para ser llevado a cabo en la formación preliminar y permanente respecto a la integración de las TIC. Dicho modelo es el Technological Pedagogical Content Knowledge (TPACK) (Mishra y Koehler, 2006). 

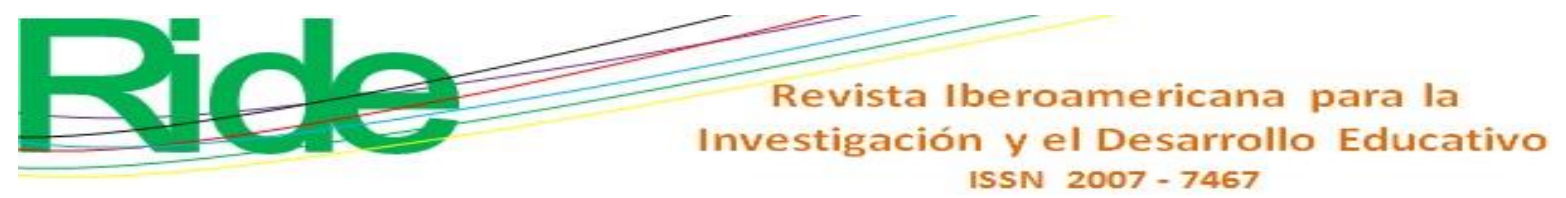

Este modelo admite comprender y puntualizar los tipos de conocimientos que requiere un profesor y las destrezas necesarias para la integración efectiva de la tecnología. Este modelo se refiere a los siguientes conceptos:

a) Technology. Representa el conocimiento técnico, la destreza de utilizar software y aplicaciones.

b) Content knowledge. Conocimiento del contenido, el cual incluye lo que se conoce de la materia o área que se enseña. Lo que el profesor imparte a los estudiantes.

c) Pedagogical knowledge. Conocimiento pedagógico, el cual se refiere a cómo enseñar.

La integración de estos tres prototipos de conocimiento es indispensable para que el uso de las tecnologías en el aula sea un éxito. El docente requiere ser un experto en el contenido de la materia a su cargo, así como saber enseñar dicho contenido. El modelo arriba mencionado se presenta en la figura 1.

Figura 1. Conocimiento del contenido pedagógico tecnológico

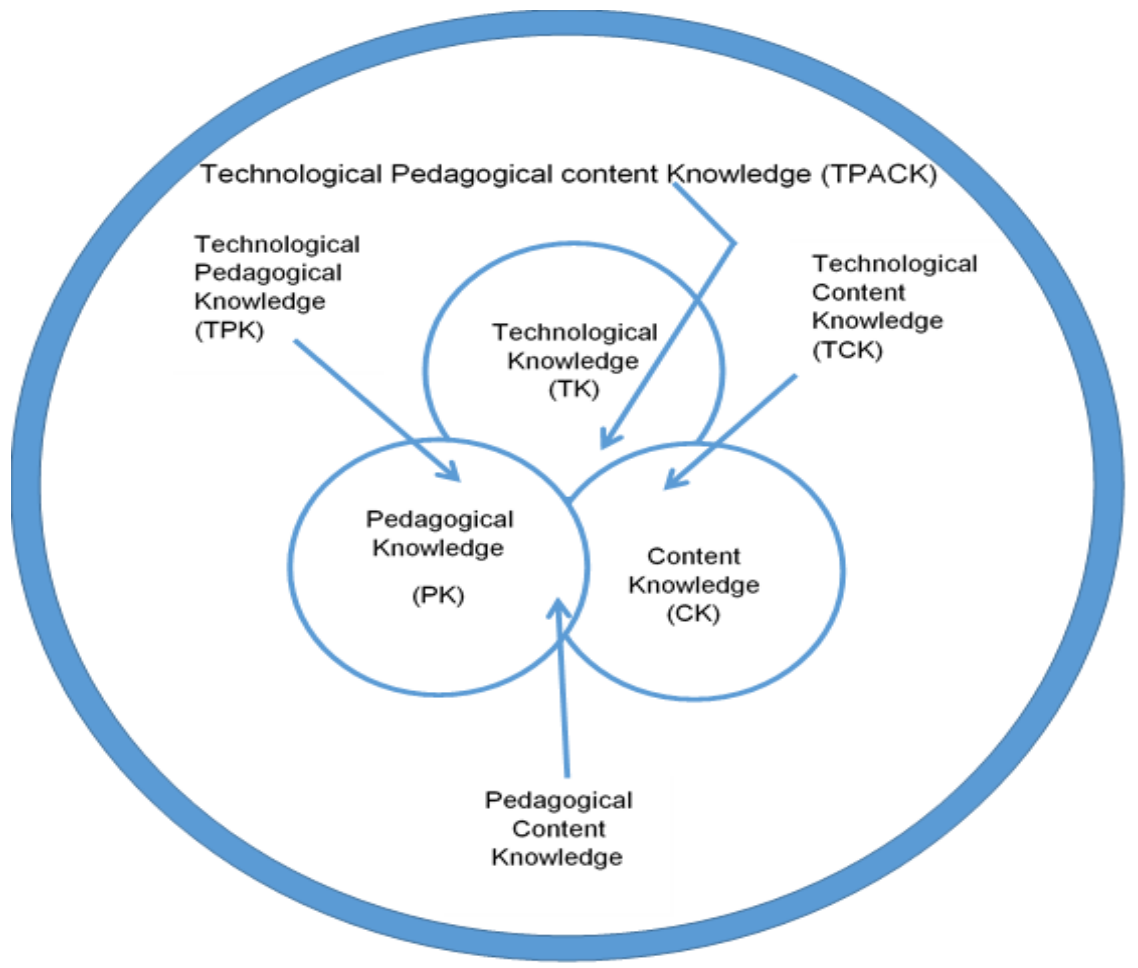

Fuente: Pico (2013, p. 72). 

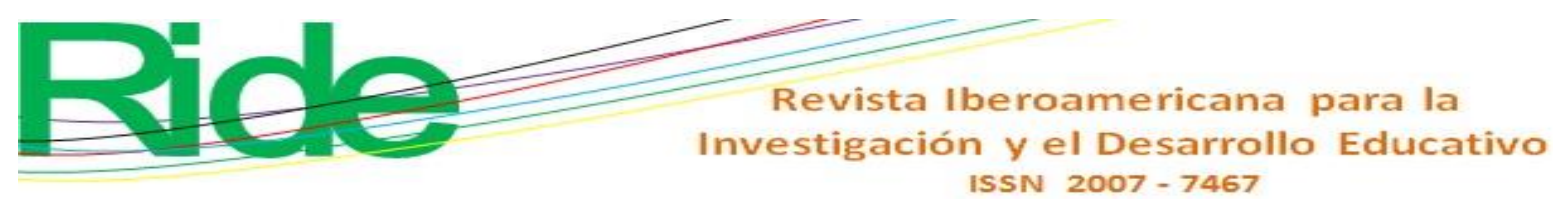

\section{Las TIC}

En esta era, el uso de las TIC ha ido en aumento. Los estudiantes deciden en qué dispositivo desean trabajar, así como en qué momento hacerlo. Es bastante cómodo traer el celular o la tableta y ponerse a trabajar en cualquier lugar, mientras haya acceso a Internet.

Las TIC también han ayudado en el proceso de aprendizaje de los estudiantes, pues, gracias a ellas, existen recursos educativos digitales que permiten tanto al docente como al estudiante realizar ejercicios, tareas o proyectos. Se puede trabajar inclusive por equipo gracias a la herramienta de Google Docs.

La universidad debe estar prevenida para encarar los cambios radicales que caracterizan a la sociedad de la información, según Sangrá y González (2004). Los profesores necesitan adaptarse a un nuevo proceso de enseñanza-aprendizaje influido por las TIC. Sin embargo, aunque las universidades doten de recursos (computadoras y acceso a Internet) a sus facultades o centros educativos, como lo menciona Tejedor (2006), "la mera dotación de recursos no es suficiente para se produzca una verdadera integración de las TIC's en la práctica escolar” (p. 21).

Por otro lado, Cabrol y Severin (2010) consideran a las TIC como una innovación disruptiva, en la medida en que obligan al cambio de las prácticas docentes, los proyectos institucionales de las escuelas y las políticas educativas. En ese sentido, Moreira, Salvat y García (2008) proponen que los objetivos de la alfabetización digital deberían dirigirse a todos.

Con el término alfabetización digital se percibe que puede haber docentes con necesidad de "alfabetizarse". Es por ello por lo que existe ese gran desafío de entender y favorecer los knowmads de la sociedad 3.0, lo cual propone Moravec (2013). Dicho término, knowmads, se refiere a los nómadas del conocimiento y la innovación.

El desafío es complejo para algunos docentes y seguirá resultando complejo con el rápido avance de la tecnología, ya que, como lo mencionan Cassany y Castellà (2011), la literacidad crítica es la capacidad de poder leer y comprender la ideología de los mensajes digitales, de poder escribir y producir textos propios con base en un análisis crítico. 


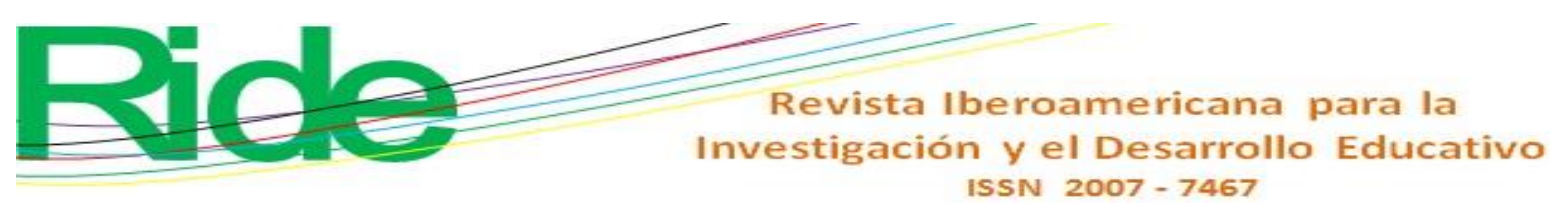

\section{Estrategias de aprendizaje y las TIC}

Necuzzi (2013) señala que las TIC han impactado en otros aspectos de los estudiantes como son la motivación, la alfabetización digital y las destrezas transversales. Por ello se debe dar importancia a conocer estas herramientas para poder usarlas en clase y así modificar la dinámica en la misma. Es decir, se debe romper el paradigma existente y dejar a los estudiantes que hagan uso de las TIC tanto para aprender como para generar conocimiento.

Las estrategias de aprendizaje también se están modificando actualmente, ya que, debido a la modernización del modelo educativo, se tiene que repercutir de alguna manera para que los profesores tanto a nivel medio superior como superior modifiquen su actuar pedagógico. Los estudiantes de nivel secundaria están aprendiendo de una forma diferente gracias a las TIC; ellos nacieron con la tecnología en puerta y desde pequeños saben manipular un teléfono móvil, lo que hace que su comportamiento en clases sea diferente, por ejemplo, ya no toman apuntes, toman fotografías con su celular. Para ellos, esto es más cómodo, práctico y fácil. Inclusive las tareas ya se envían por WhatsApp al jefe de grupo y este se encarga de avisarles a todos.

Las TIC se han venido utilizando cotidianamente por los estudiantes, lo cual implica que las competencias no evidentes resulten invisibles en los entornos formales. Y se llegan a hacer invisibles debido a que algunas son ignoradas o resultan irrelevantes dentro del círculo académico. Por otro lado, Cobo y Moravec (2011) señalan cuestiones centrales que hacen invisible a la construcción de la noción de aprendizaje.

El aprendizaje invisible también se está dando en los profesores, ya que algunos de forma individual utilizan herramientas existentes en Internet sin que asistan a un curso o capacitación de dicha herramienta, sino que la utilizan porque la consideran útil para su clase; o bien, consideran que puede ser amena, dinámica y de fácil uso para los estudiantes.

Por otra parte, Siemens (2014) nos menciona lo siguiente: "El aprendizaje es un proceso continuo, que dura toda la vida y que la tecnología está alterando (recableando) nuestros cerebros. Las herramientas que utilizamos definen y dan forma a nuestro pensamiento" (p.1). Sin embargo, desde antes que existieran las herramientas tecnológicas, el aprendizaje se tenía que adaptar a nuevas situaciones. Como lo explican Díaz y Hernández (2002): 

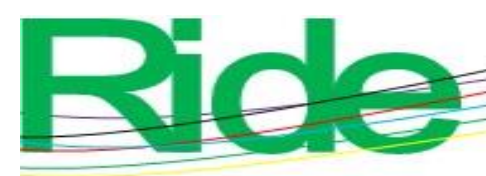

Revista Iberoamericana para la

Investigación y el Desarrollo Educativo

ISSN $2007-7467$

Aprender a aprender implica la capacidad de reflexionar en la forma en que se aprende y actuar en consecuencia, autorregulando el propio proceso de aprendizaje mediante el uso de estrategias flexibles y apropiadas que se transfieren y adaptan a nuevas situaciones (p. 12).

Allí justamente recae la importancia de conocer qué herramientas son las más utilizadas por los estudiantes hoy en día, para que los docentes también puedan seguir actualizándose, pero sobre todo para que apliquen estrategias de aprendizaje diferentes e innovadoras.

\section{Metodología}

Para este trabajo se eligió una modalidad de investigación de tipo cuantitativo. Los dos tipos de diseño metodológico que se incluyen en la metodología cuantitativa son el experimental y el no experimental. La investigación aquí mostrada está dentro de este último y es de tipo encuesta (McMillan y Schumacher, 2005). El avance tecnológico permite cuestionarse acerca de cuáles son las herramientas que más utilizan los alumnos. Si estas representan mejoras en la percepción hacia el contenido de la clase y de la efectividad misma del proceso de enseñanza-aprendizaje. El conocer ciertos indicadores sobre los cuales poner atención para la mejora educativa es de gran trascendencia.

Primero, se realizó el instrumento con ayuda de un formulario de Google. Posteriormente se envió a diversos grupos de diversas facultades, tanto a nivel licenciatura como a nivel posgrado, esto con ayuda de los profesores y con ayuda del jefe de grupo en algunos casos, ya que algunos de estos tienen correo grupal. Así, pues, se les envió la liga a ese correo y ya cada quien se encargaba de contestar la encuesta. Esto se realizó en una institución pública de educación superior.

Para las variables, en un principio, se tomaron las siguientes dimensiones: "Demografía", "Herramientas usadas en clase", "Internet y redes sociales" y, por último, "Juegos", pero más adelante no se agruparon de acuerdo a dichas dimensiones. Las variables se dejaron como aparecen en las gráficas de los resultados, es decir, se tuvo la variable para "Herramienta de textos" y se dejó así su porcentaje, de acuerdo al número de veces que aparece, aunque se puede agrupar en la dimensión de "Herramientas usadas en clase" para que exista una mejor descripción. Cabe 

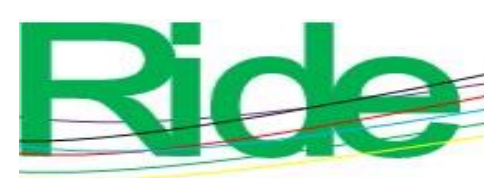

Revista Iberoamericana para la

Investigación y el Desarrollo Educativo

ISSN $2007-7467$

mencionar que el instrumento se generó de acuerdo con los programas que se manejan en esta institución.

Las variables a utilizar fueron las siguientes: "Herramientas estudiantiles", "Herramientas para uso personal", "Herramientas para correo y navegadores" y "Herramientas para juego".

Para recabar la información, se tomó del formulario de Google la hoja electrónica con los datos recabados en las encuestas. Se bajó como hoja de Excel y, a partir de ahí, se comenzó a trabajar en dicho programa para la realización de los porcentajes y las gráficas. Se realizaron tablas dinámicas y sus respectivas gráficas. Cabe mencionar que se realizaron gráficas propias, no se usaron las que el formulario de Google genera automáticamente.

\section{Población y muestra}

Se realizó un diseño cuantitativo-descriptivo con un análisis comparativo entre las herramientas más utilizadas por los estudiantes. A partir de allí se comprendió el uso tanto para la escuela como para uso personal. Se tomó en consideración una población de 1161 estudiantes de diversas facultades de una institución de educación superior, y la muestra resultante fue de 224 alumnos de diversos semestres, tanto de licenciatura como de posgrado. Cabe señalar que hubo mayor participación de algunas facultades sobre otras, pero no se sabe el motivo, simplemente fueron más los de ciertas facultades quienes contestaron el cuestionario en línea en comparación con los de otras (posiblemente pudo ser el horario en que se les envió, que fue por la tarde, pero no creemos que realmente eso haya influido).

\section{Instrumento}

Para recabar la información se utilizó la técnica de evaluación por encuestas. Y el instrumento utilizado para este trabajo fue una encuesta electrónica en línea con ayuda de un formulario de Google Docs.

Las respuestas se fueron agrupando y analizando, dependiendo del rubro al que pertenecían. Por ejemplo, "Herramientas para uso personal", "Herramientas electrónicas utilizadas en la escuela", o bien "Plataformas utilizadas", "Antivirus" y "Correo más utilizado" por dichos estudiantes. Se calculó el porcentaje para todos los casos utilizando la hoja de Microsoft Excel.

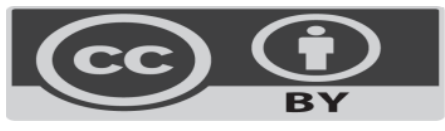



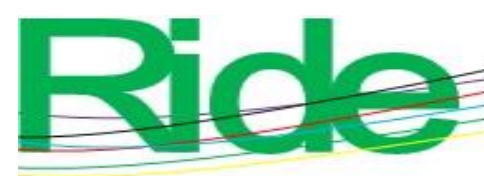

\section{Revista Iberoamericana para la Investigación y el Desarrollo Educativo ISSN $2007-7467$}

\section{Resultados}

La tabla 1 muestra los porcentajes de la representación de las facultades que participaron en este trabajo. La que tuvo mayor participación fue la de Química.

Tabla 1. Facultades participantes

\begin{tabular}{|l|r|}
\hline Facultad en la que te & Porcentaje \\
\hline Oncuentras & $0 \%$ \\
\hline Informática & $1 \%$ \\
\hline Ingeniería & $3 \%$ \\
\hline Contaduría y Administración & $4 \%$ \\
\hline Química & $92 \%$ \\
\hline
\end{tabular}

Fuente: Elaboración propia

La figura 2 muestra que hubo mayor participación de la Facultad de Química, siguiendo la Facultad de Contaduría y Administración, y no muy lejos de ella la Facultad de Ingeniería.

Figura 2. Gráfica que muestra la participación de los estudiantes por facultad

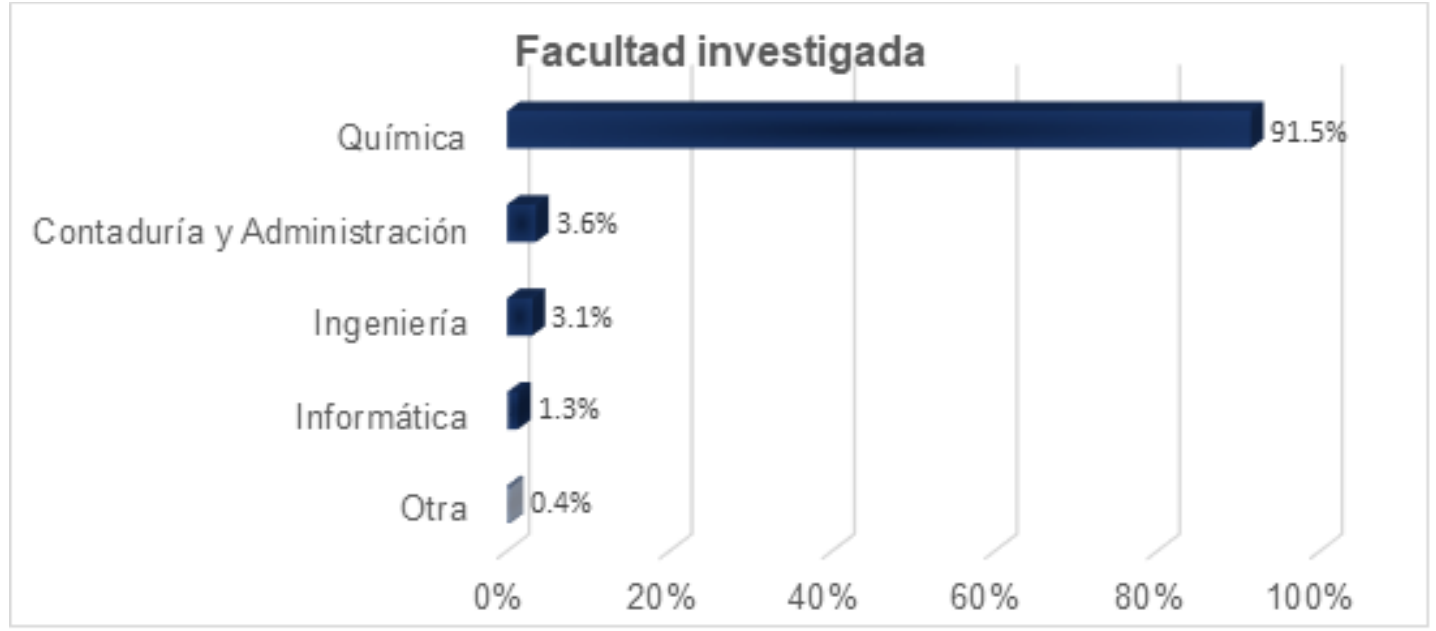

Fuente: Elaboración propia 

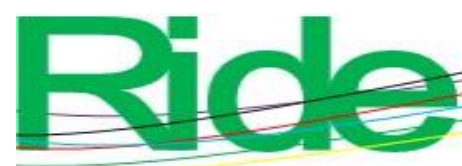

\section{Revista Iberoamericana para la Investigación y el Desarrollo Educativo ISSN $2007-7467$}

A continuación, la tabla 2 muestra los porcentajes de los semestres que participaron en este trabajo de investigación. Se puede apreciar que el tercer semestre fue el que mayor participación tuvo.

Tabla 2. Semestres que participaron

\begin{tabular}{|l|r|}
\hline Semestre & Porcentaje \\
\hline $3 .^{\circ}$ & $21 \%$ \\
\hline $1 .^{\circ}$ & $16 \%$ \\
\hline $7 .^{\circ}$ & $13 \%$ \\
\hline $9 .^{\circ}$ & $13 \%$ \\
\hline $5 .^{\circ}$ & $10 \%$ \\
\hline $8 .^{\circ}$ & $8 \%$ \\
\hline $4 .^{\circ}$ & $5 \%$ \\
\hline $6 .{ }^{\circ}$ & $5 \%$ \\
\hline $2 .^{\circ}$ & $3 \%$ \\
\hline Maestría & $3 \%$ \\
\hline Doctorado & $1 \%$ \\
\hline $10 .{ }^{\circ}$ & $0 \%$ \\
\hline Especialidad & $0 \%$ \\
\hline \multicolumn{2}{|l|}{ Fuente: Elaboración propia }
\end{tabular}

La figura 3 detalla que el semestre con mayor participación fue el de tercero, y detrás suyo el primero, sin dejar de mencionar la participación del área de posgrado. 

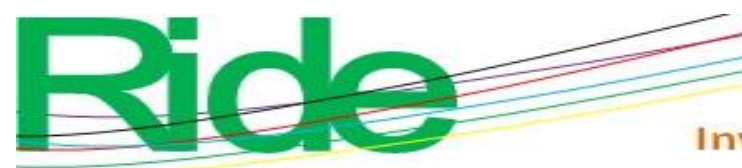

Revista Iberoamericana para la

Investigación y el Desarrollo Educativo

ISSN $2007-7467$

Figura 3. Gráfica de estudiantes que participaron por semestre

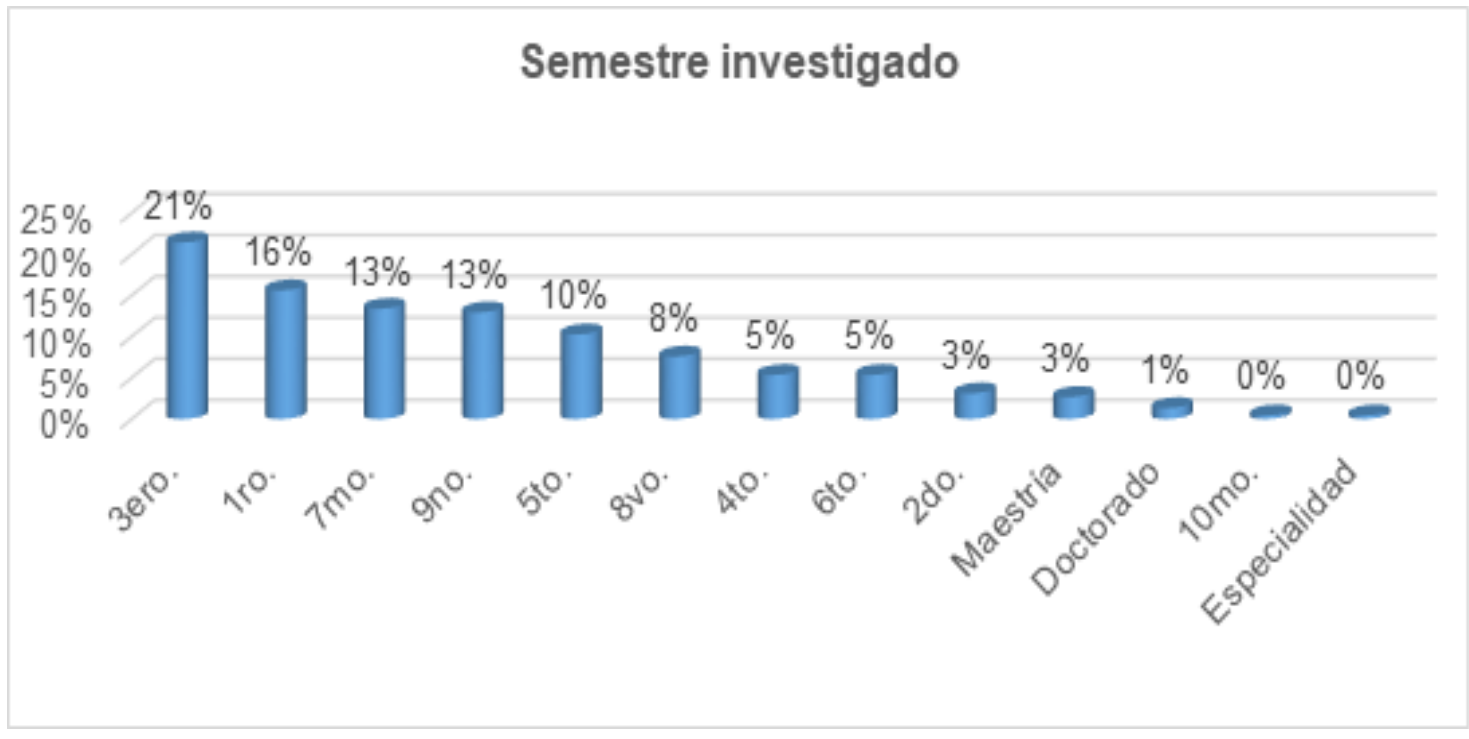

Fuente: Elaboración propia

En la figura 4 se muestra que la participación femenina fue significativamente mayor que la participación masculina.

Figura 4. Gráfica de participación por género

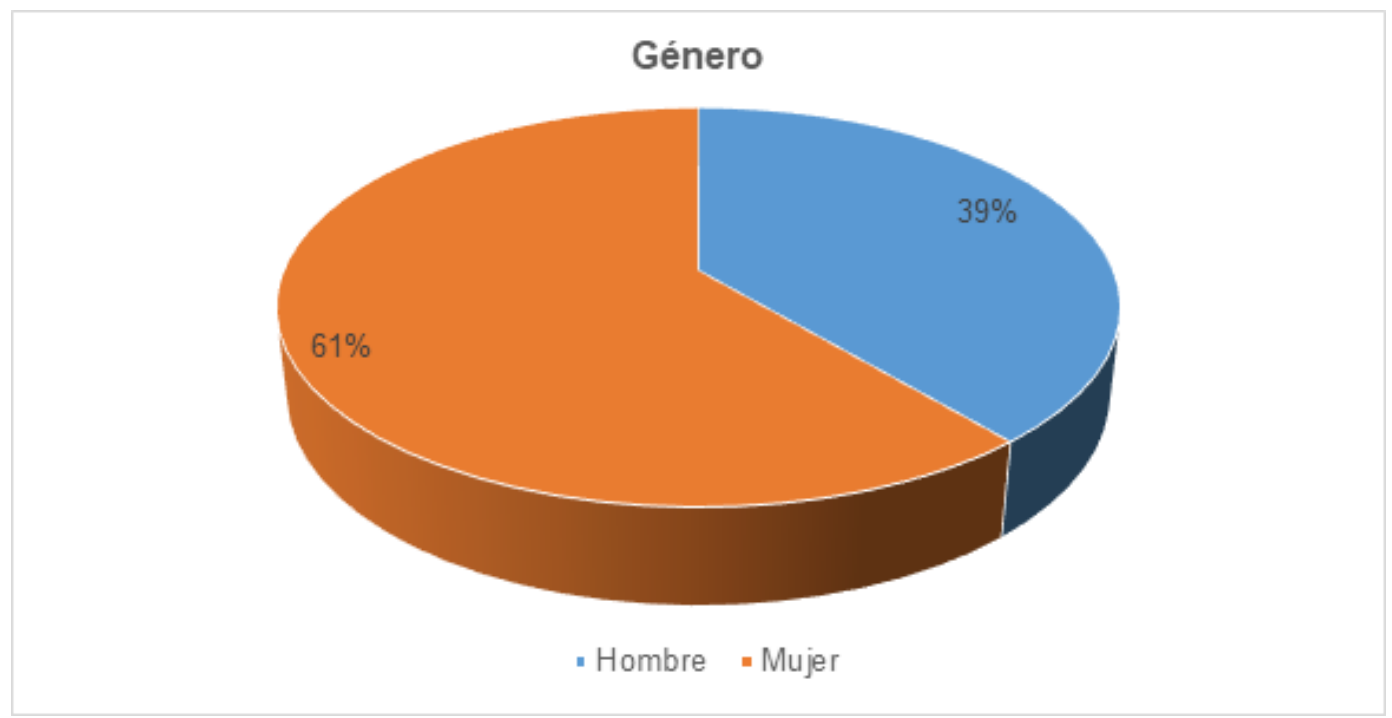

Fuente: Elaboración propia 


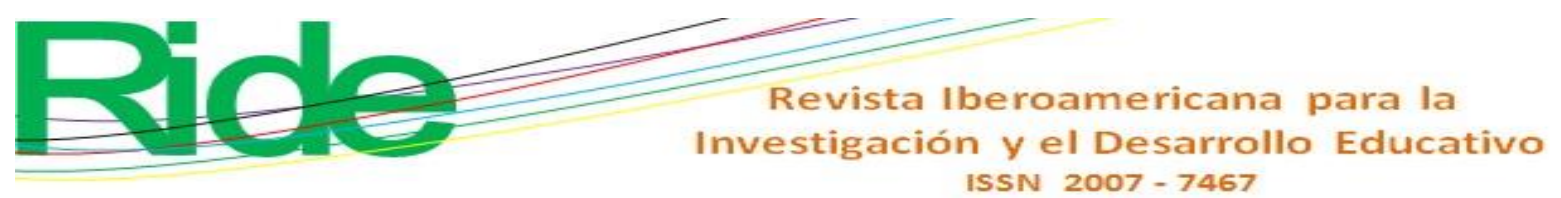

En la figura 5 se muestra la participación por edades de los estudiantes. El rango con mayor representación fue el de 21-23 años, siguiéndole el de 18-20 años.

Figura 5. Gráfica de participación por edad de los estudiantes

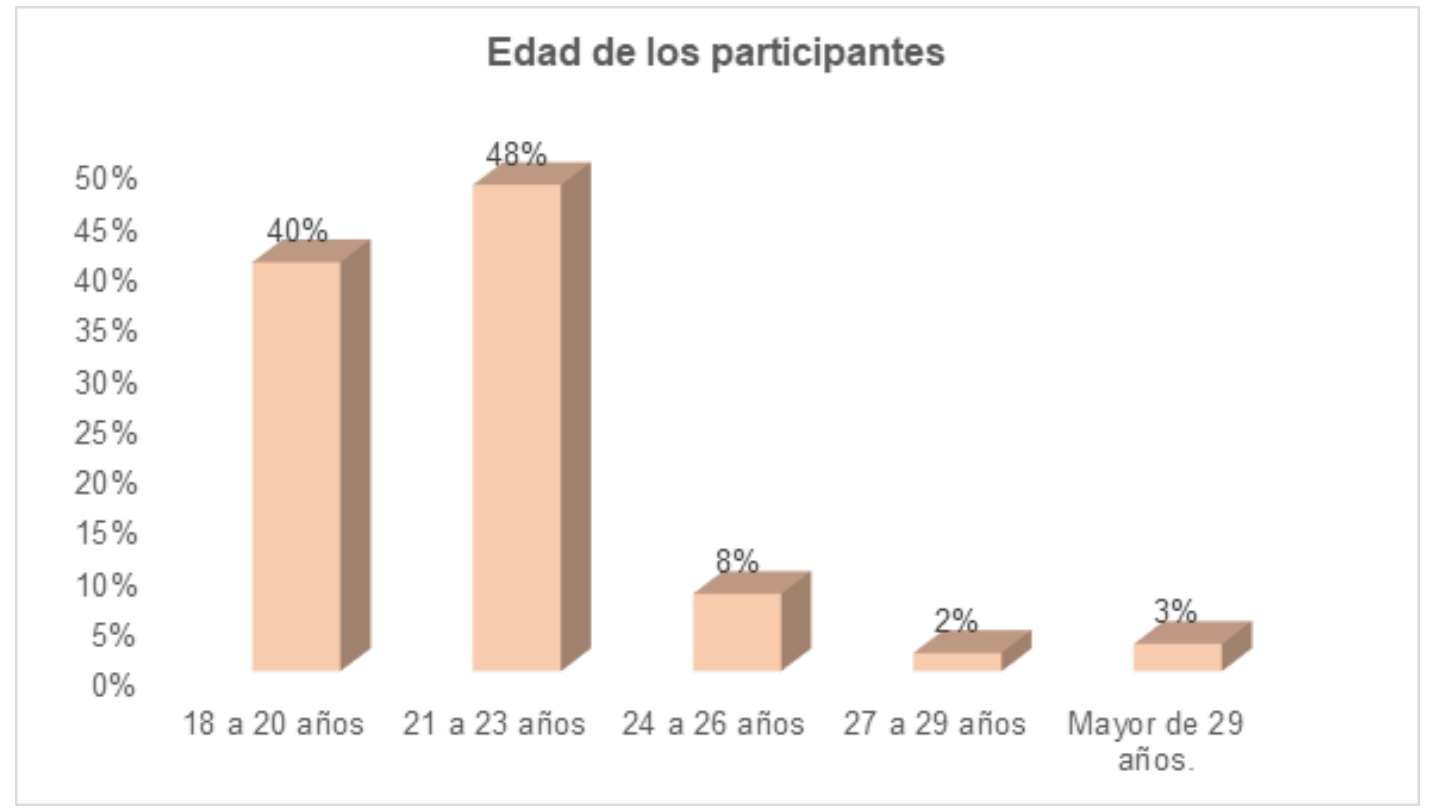

Fuente: Elaboración propia

La figura 6 detalla el correo que más utilizan los estudiantes. Aquí sobresale Gmail, seguido por el servicio de Hotmail. Es de destacar que el correo institucional casi no se utiliza. 

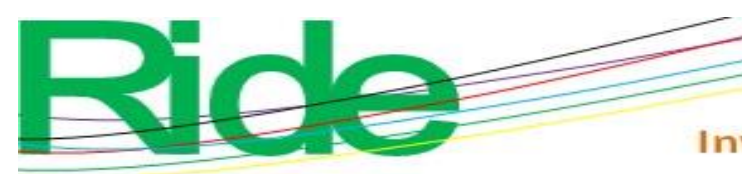

Revista Iberoamericana para la Investigación y el Desarrollo Educativo ISSN $2007-7467$

Figura 6. Gráfica de correo que más utilizan los estudiantes

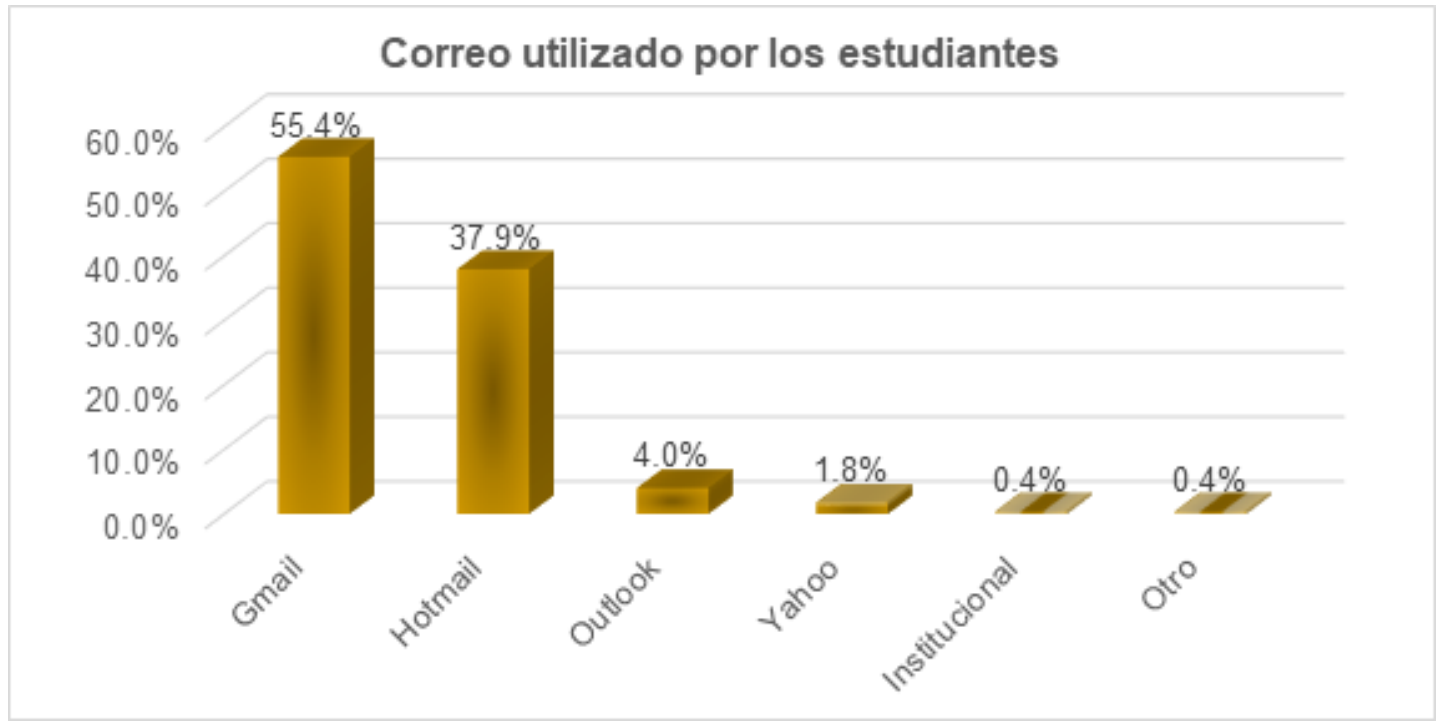

Fuente: Elaboración propia

En la figura 7 se muestra que el procesador de textos Microsoft Word es el más utilizado por los estudiantes.

Figura 7. Gráfica de procesador de textos más utilizado

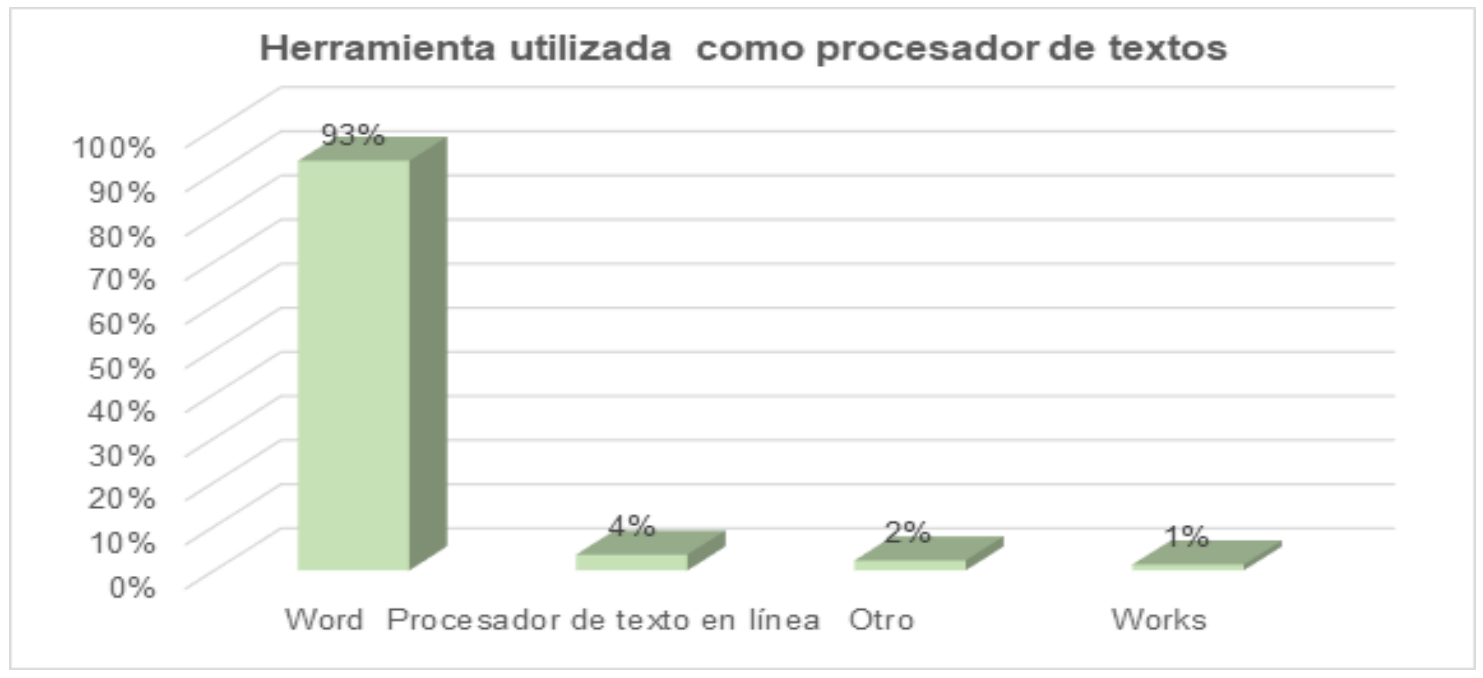

Fuente: Elaboración propia

En la figura 8 se detalla la herramienta que más se utiliza para archivos PDF: Adobe Acrobat es el que sobresale, seguido por Nitro Pro. 

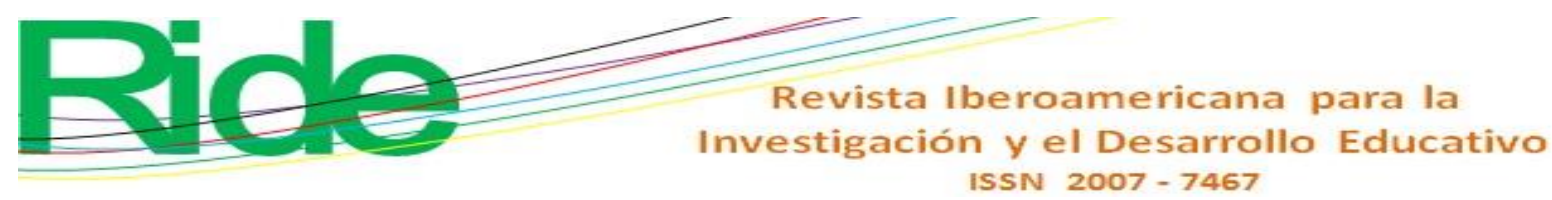

Figura 8. Herramienta más utilizada por los estudiantes para archivos PDF

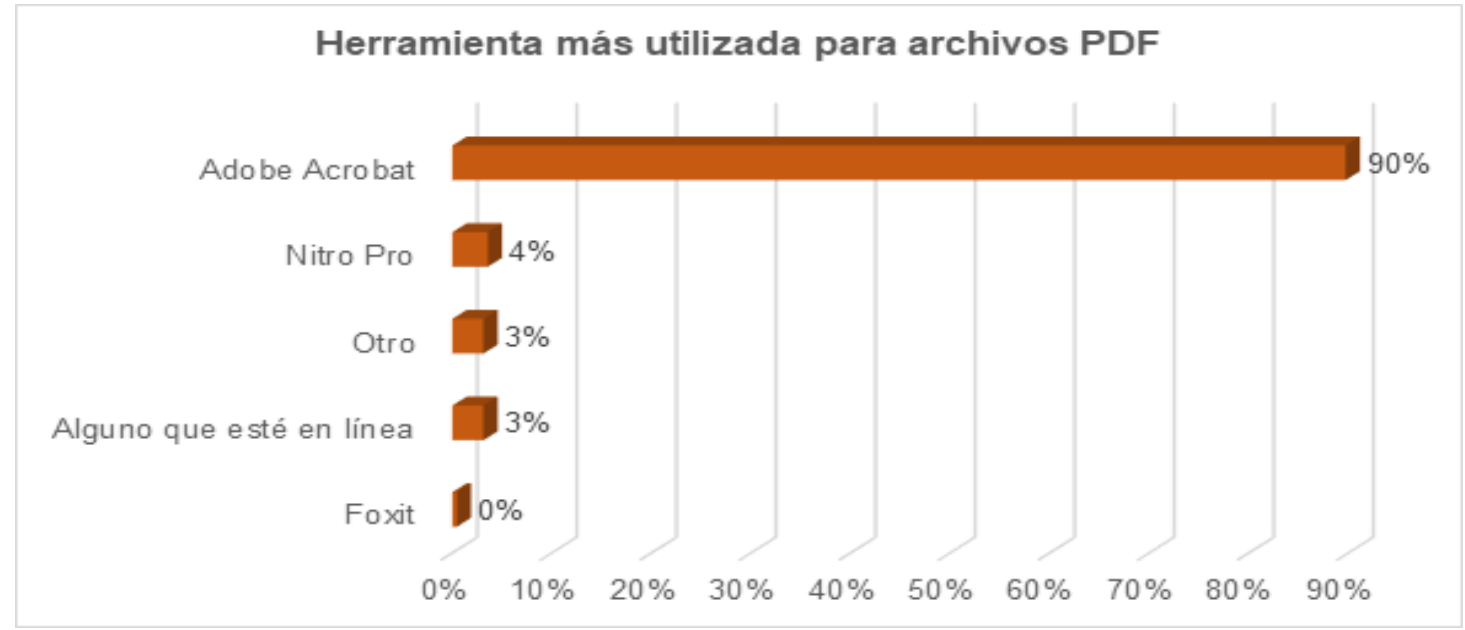

Fuente: Elaboración propia

La figura 9, por su parte, muestra los navegadores que utilizan los estudiantes con mayor asiduidad. Google Chrome protagoniza este rubro con mucha diferencia sobre Mozilla, que es el segundo.

Figura 9. Herramienta de navegadores de internet que más utilizan los estudiantes

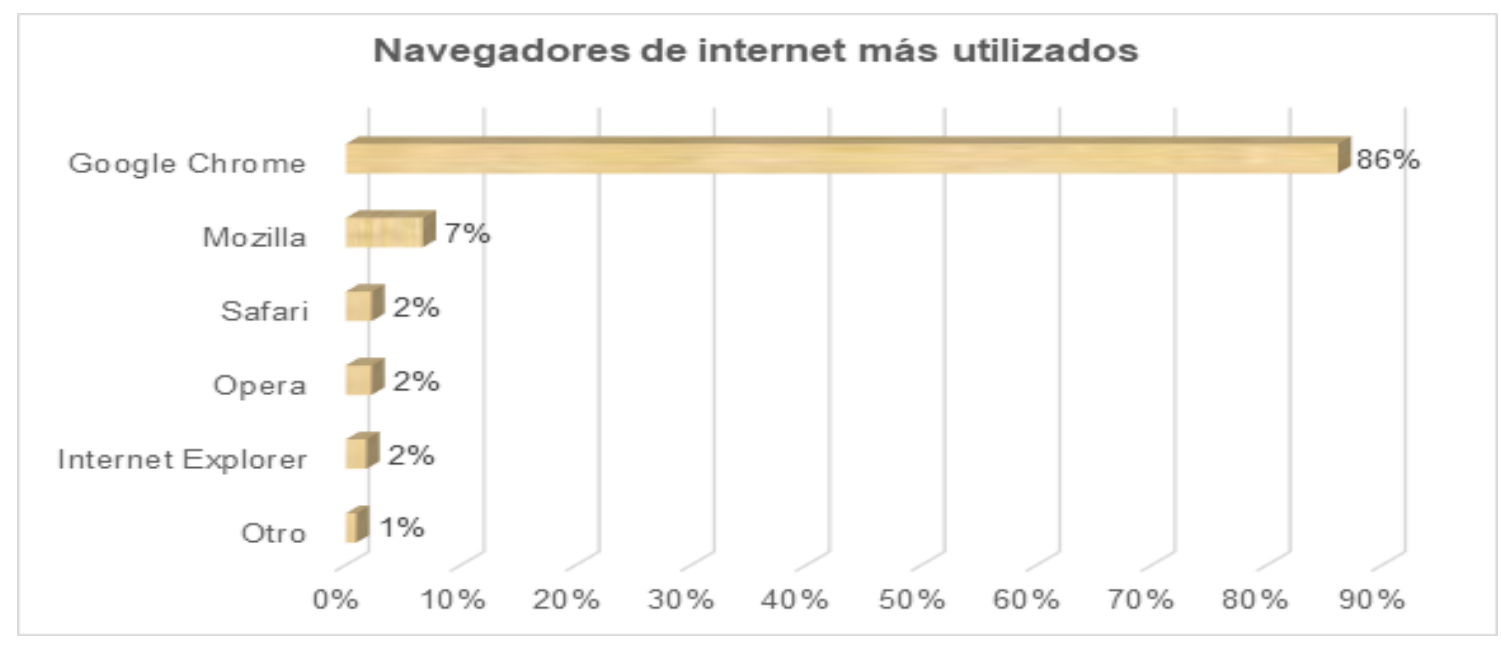

Fuente: Elaboración propia

La figura 10 detalla que el buscador web que despunta y por mucho es el de Google. A pesar de que existen más buscadores, no son utilizados por los estudiantes. 

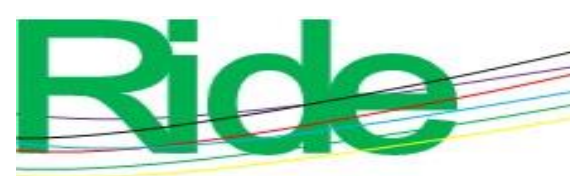

\section{Revista Iberoamericana para la Investigación y el Desarrollo Educativo ISSN $2007-7467$}

Figura 10. Buscadores de internet utilizados por los estudiantes

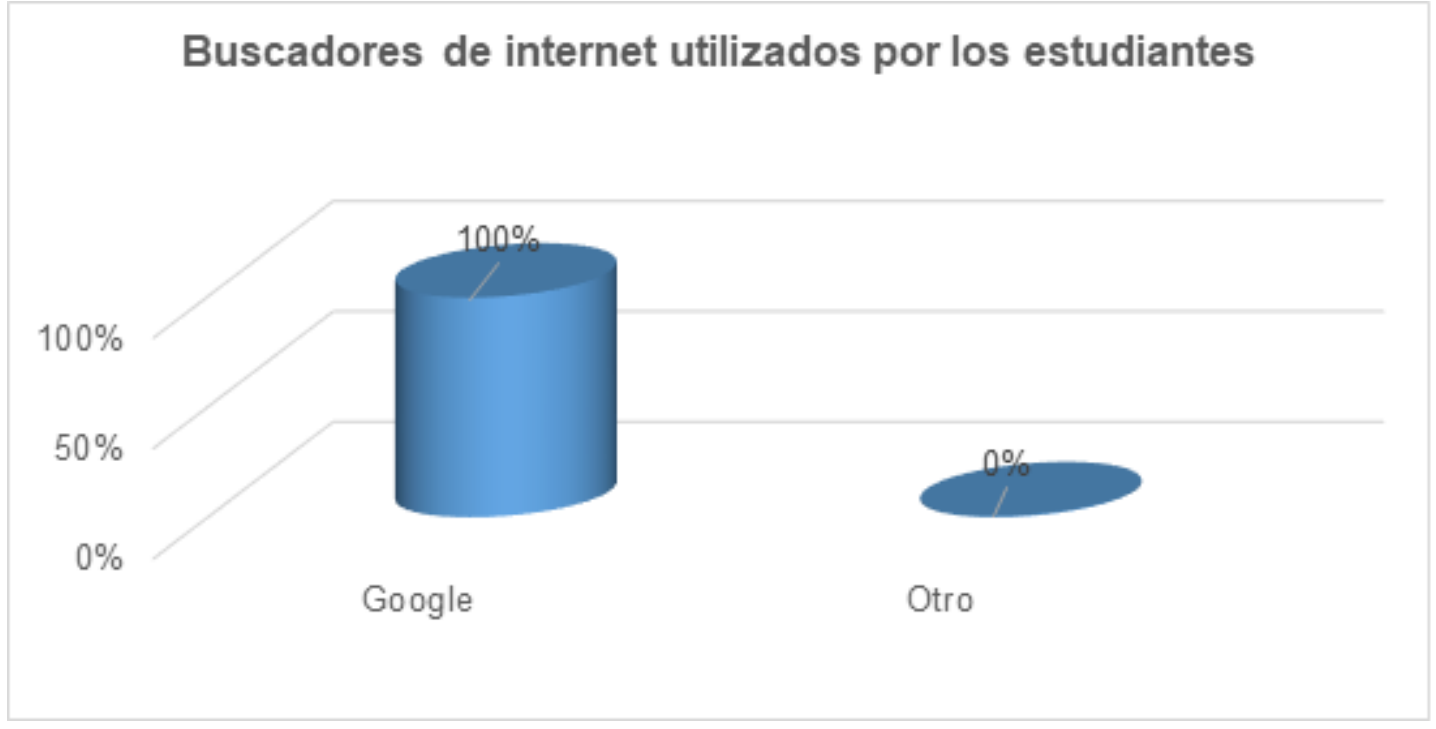

Fuente: Elaboración propia

En la tabla 3 se muestra la herramienta que más prefieren los estudiantes para sus clases, donde sobresale la de powerpoint con un alto porcentaje.

Tabla 3. Porcentaje de herramientas más utilizadas para presentaciones

\begin{tabular}{|l|r|}
\hline $\begin{array}{l}\text { Herramienta que más utilizas } \\
\text { para presentaciones en tus clases }\end{array}$ & Porcentaje \\
\hline Emaze & $0 \%$ \\
\hline Zoho Presentation & $0 \%$ \\
\hline Brainshark & $0 \%$ \\
\hline Knovio & $0 \%$ \\
\hline Slides & $3 \%$ \\
\hline PowToon & $3 \%$ \\
\hline Otra & $4 \%$ \\
\hline Prezzi & $16 \%$ \\
\hline PowerPoint & $95 \%$ \\
\hline
\end{tabular}

Fuente: Elaboración propia 

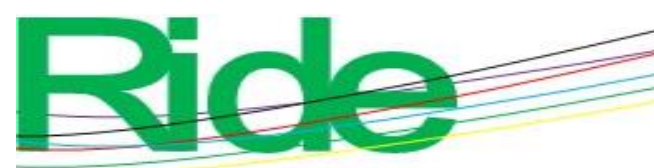

\section{Revista Iberoamericana para la Investigación y el Desarrollo Educativo ISSN $2007-7467$}

En la figura 11 se puntualiza que la herramienta más utilizada en las presentaciones es Microsoft PowerPoint, a pesar de que existen otras muy dinámicas, como la de PowToon.

Figura 11. Herramienta utilizada por los estudiantes para las presentaciones

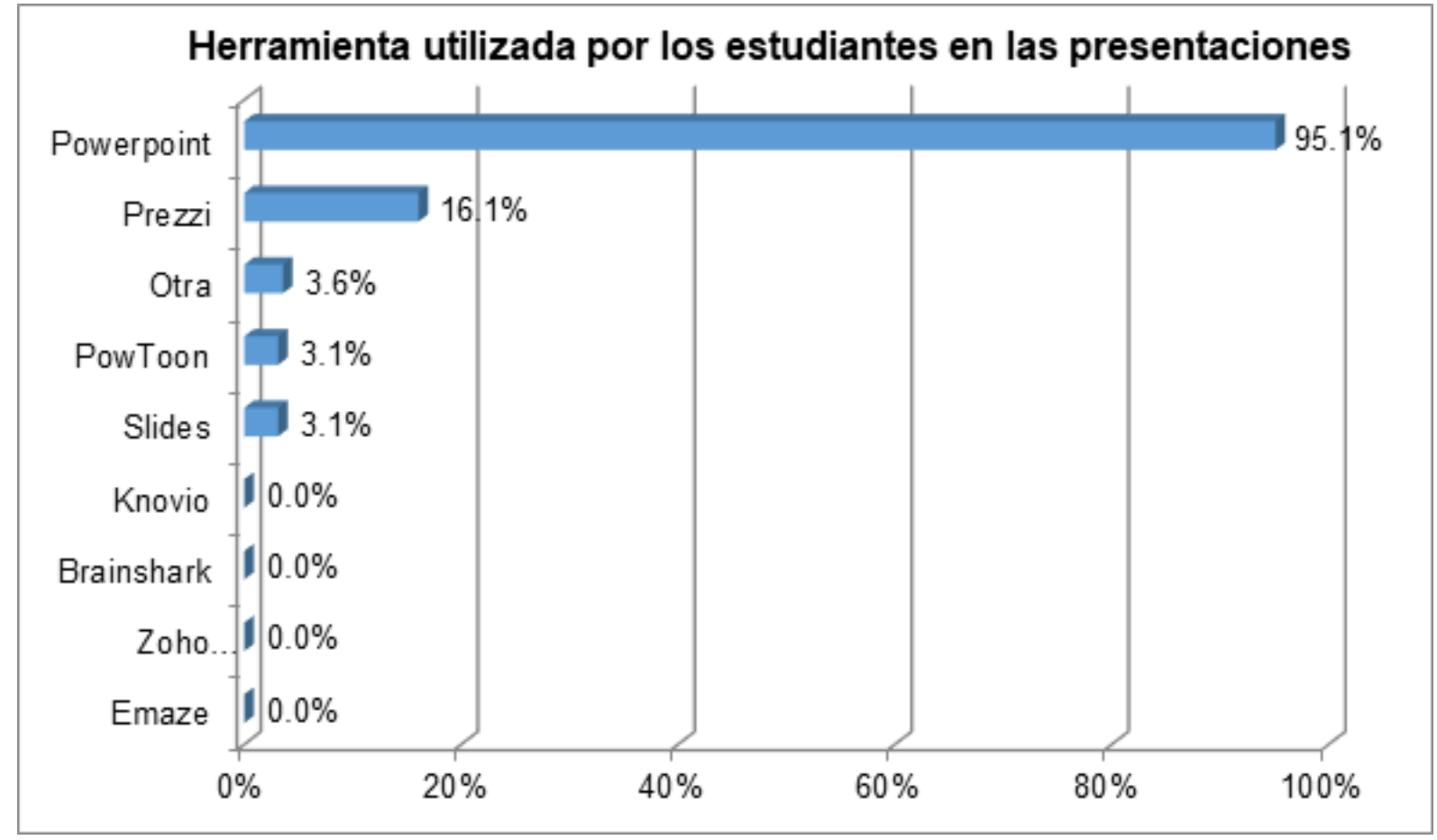

Fuente: Elaboración propia

En la figura 12, mientras tanto, se detalla que la herramienta más sobresaliente para ejercicios estadísticos es la de Excel, seguido por la de Minitab. 

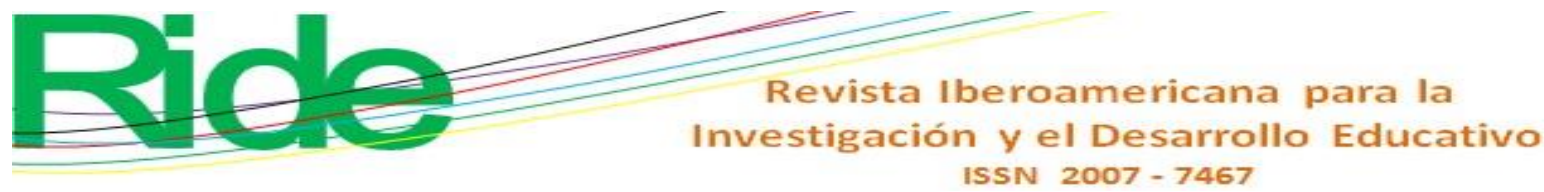

Figura 12. Herramienta más utilizada por los estudiantes para ejercicios estadísticos

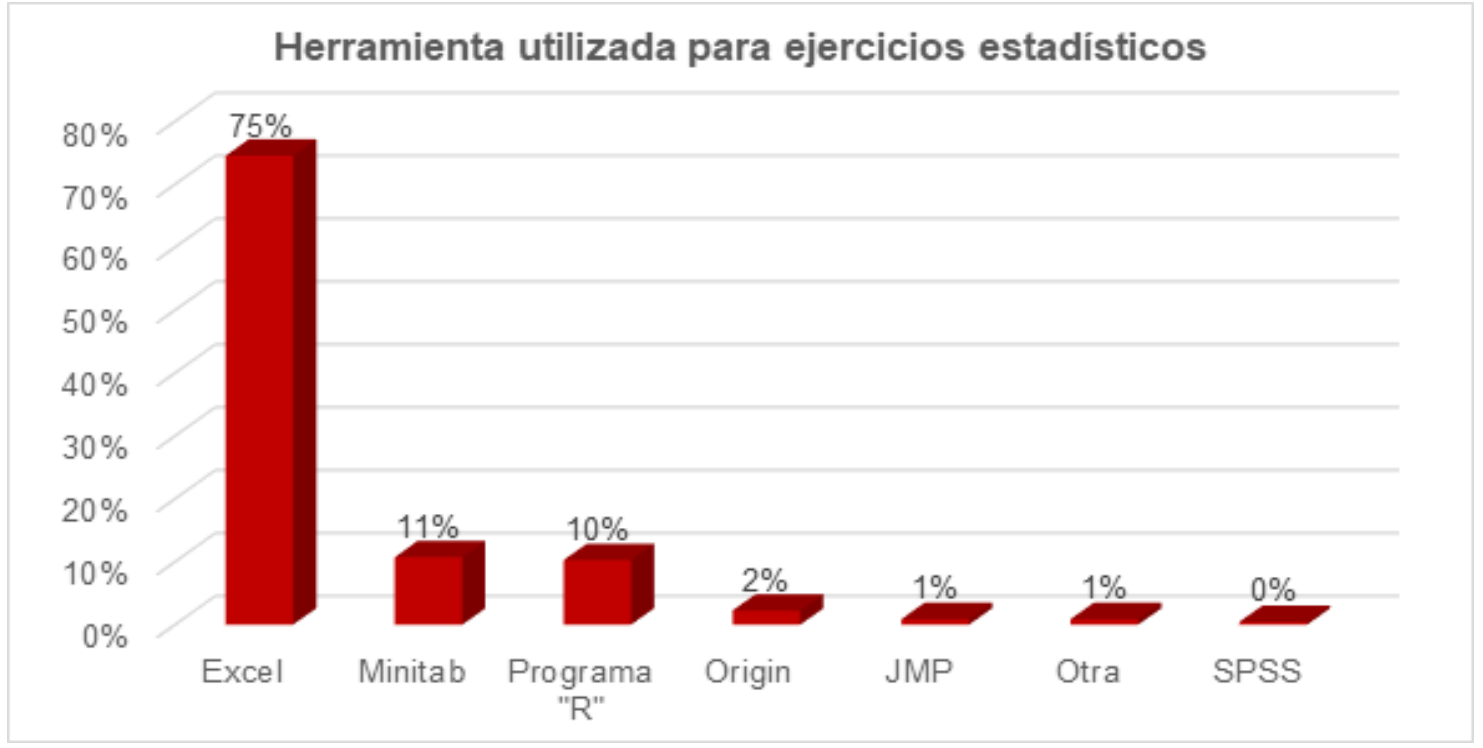

Fuente: Elaboración propia

La figura 13 especifica que el antivirus más utilizado por los estudiantes es Avast; Norton y McAfee se posicionaron en segundo y tercer lugar respectivamente.

Figura 13. Antivirus más utilizado por los estudiantes

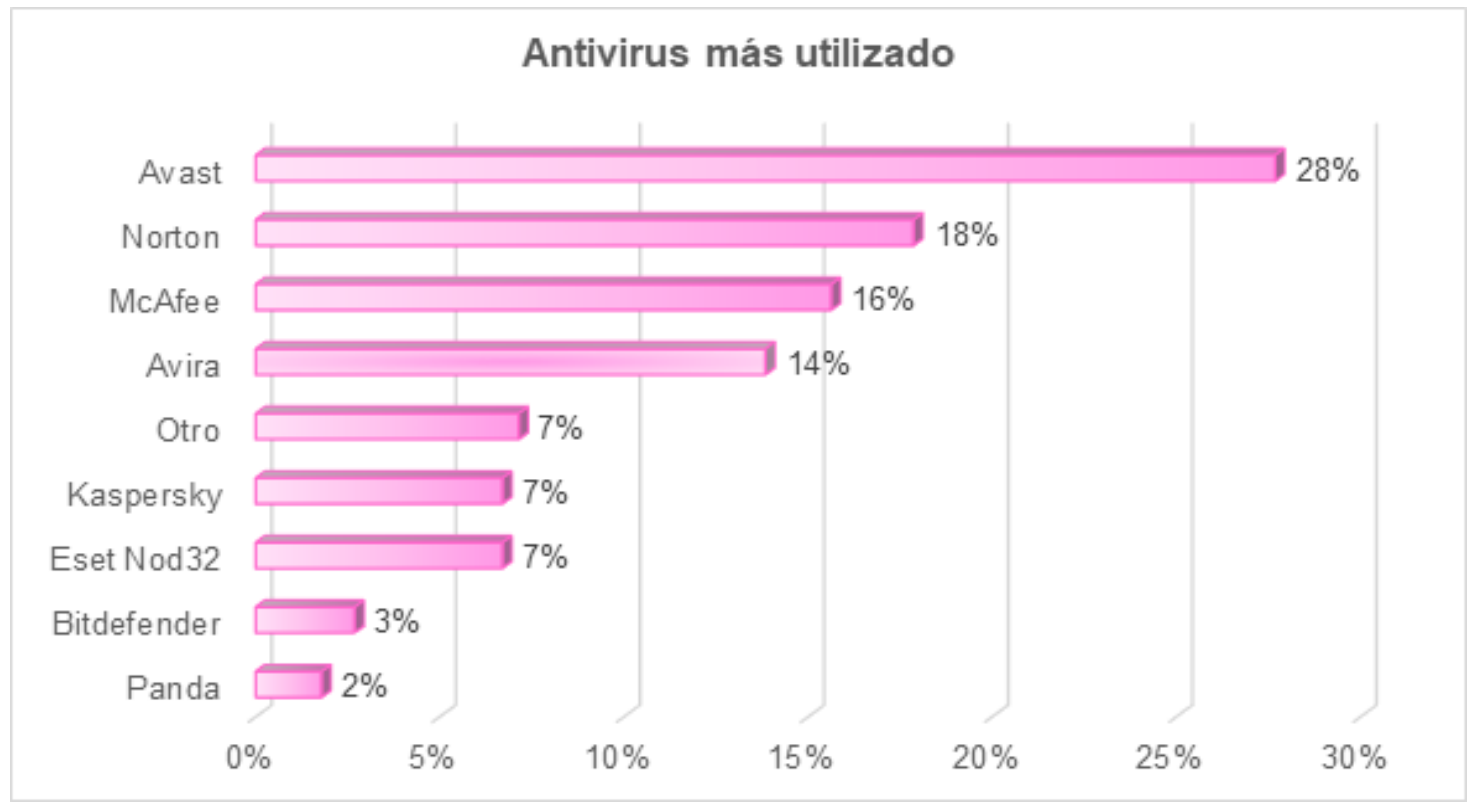

Fuente: Elaboración propia 

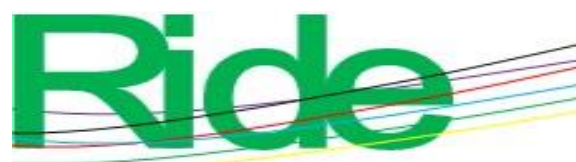

\section{Revista Iberoamericana para la Investigación y el Desarrollo Educativo ISSN $2007-7467$}

En la figura 14 sobresale Facebook como la red social más utilizada, seguida de Youtube.

Figura 14. Redes sociales utilizadas por los estudiantes

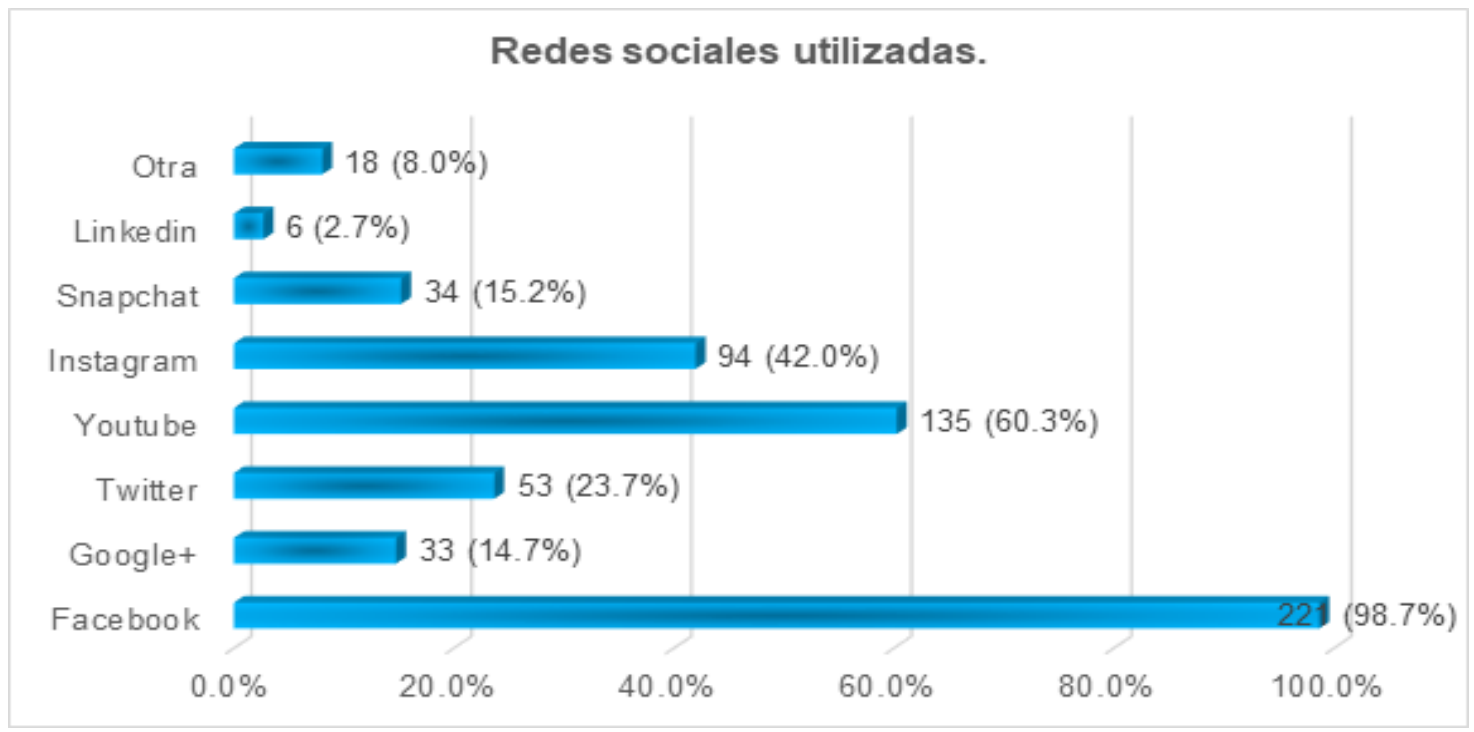

Fuente: Elaboración propia

La figura 15 muestra que la herramienta más utilizada para chatear es WhatsApp, seguida por Messenger de Facebook.

Figura 15. Herramientas utilizadas por los estudiantes para chatear Herramientas utilizadas para chatear.

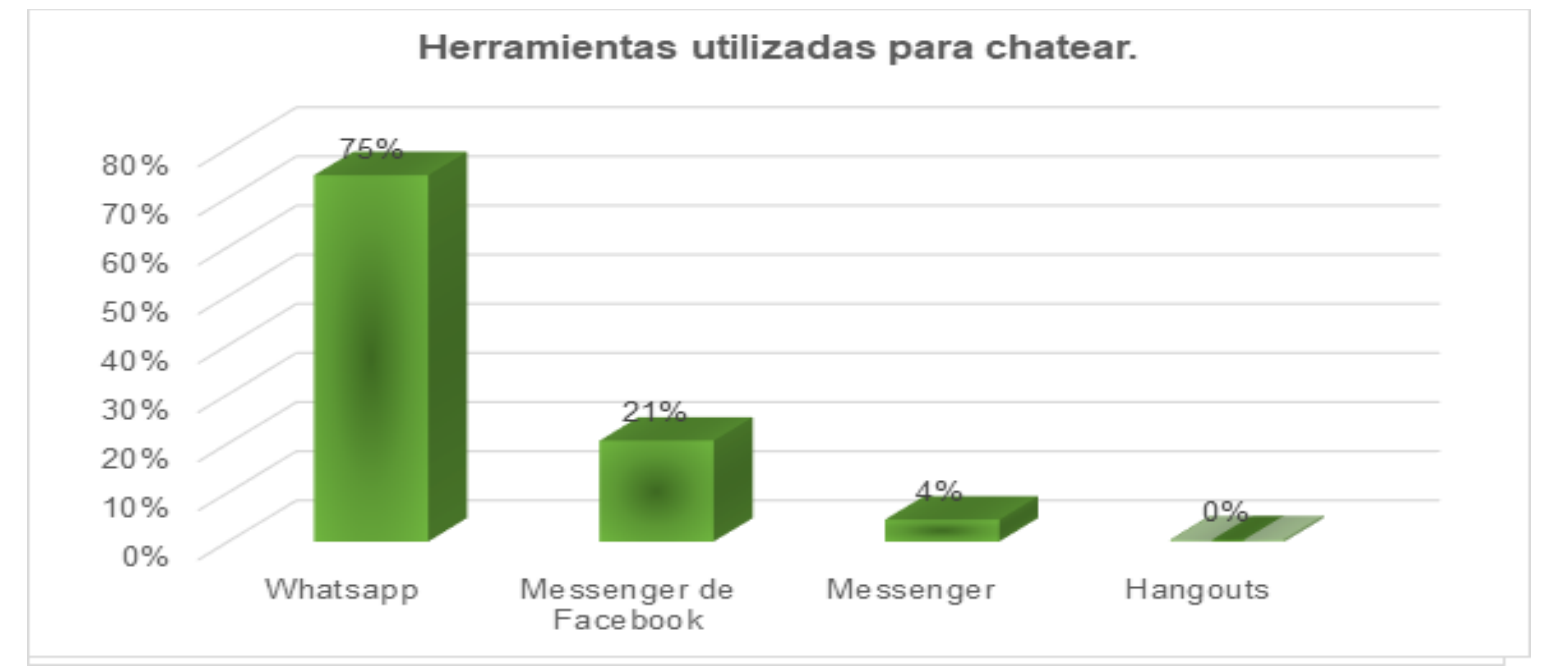

Fuente: Elaboración propia 

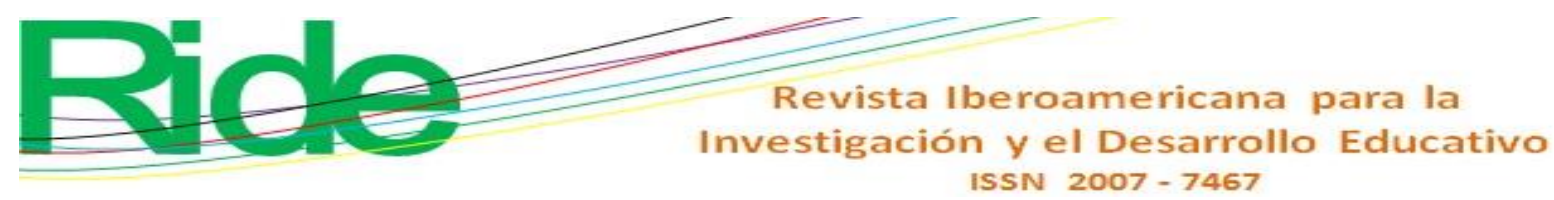

La figura 16 muestra si los estudiantes han utilizado o no videoconferencia en sus clases. Sobresale que la mayoría no la ha utilizado. En efecto, solo $7 \%$ la ha utilizado.

Figura 16. Videoconferencia utilizada en clases por los estudiantes

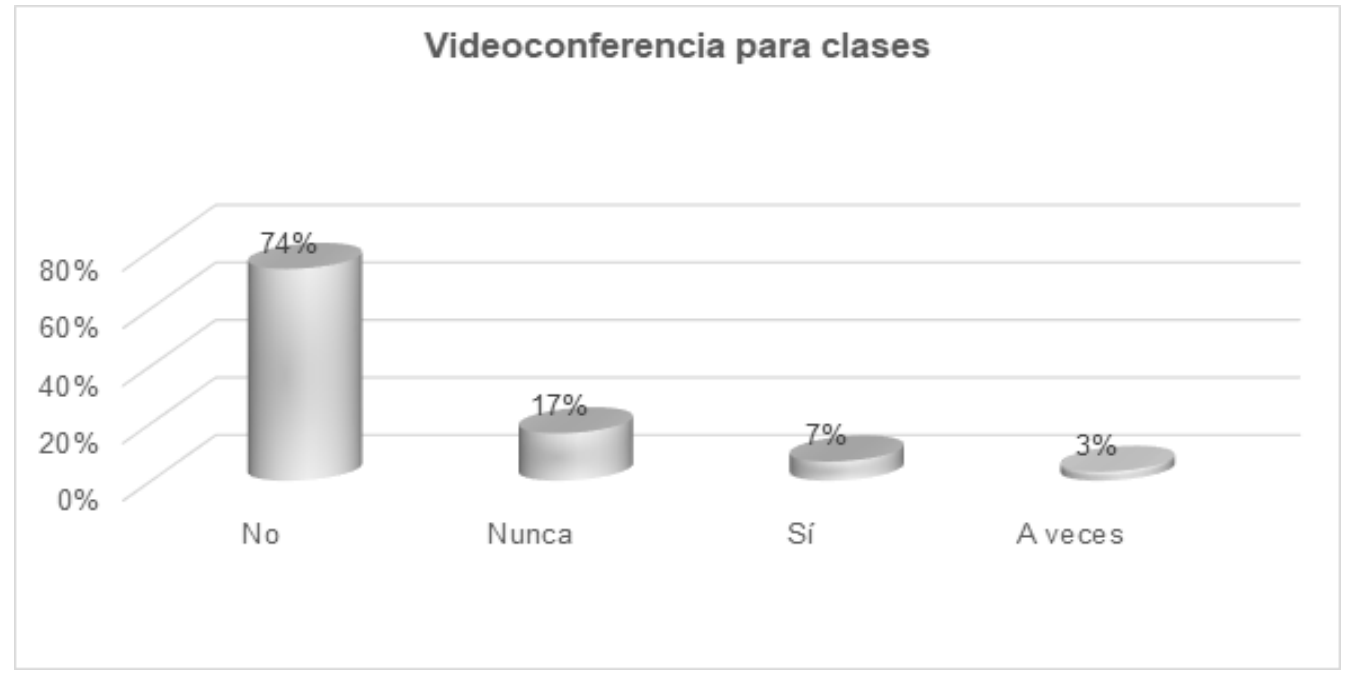

Fuente: Elaboración propia

La figura 17 especifica el programa que utilizan los estudiantes para videoconferencia. Skype se coloca en primer lugar.

Figura 17. Software utilizado para videoconferencia por los estudiantes

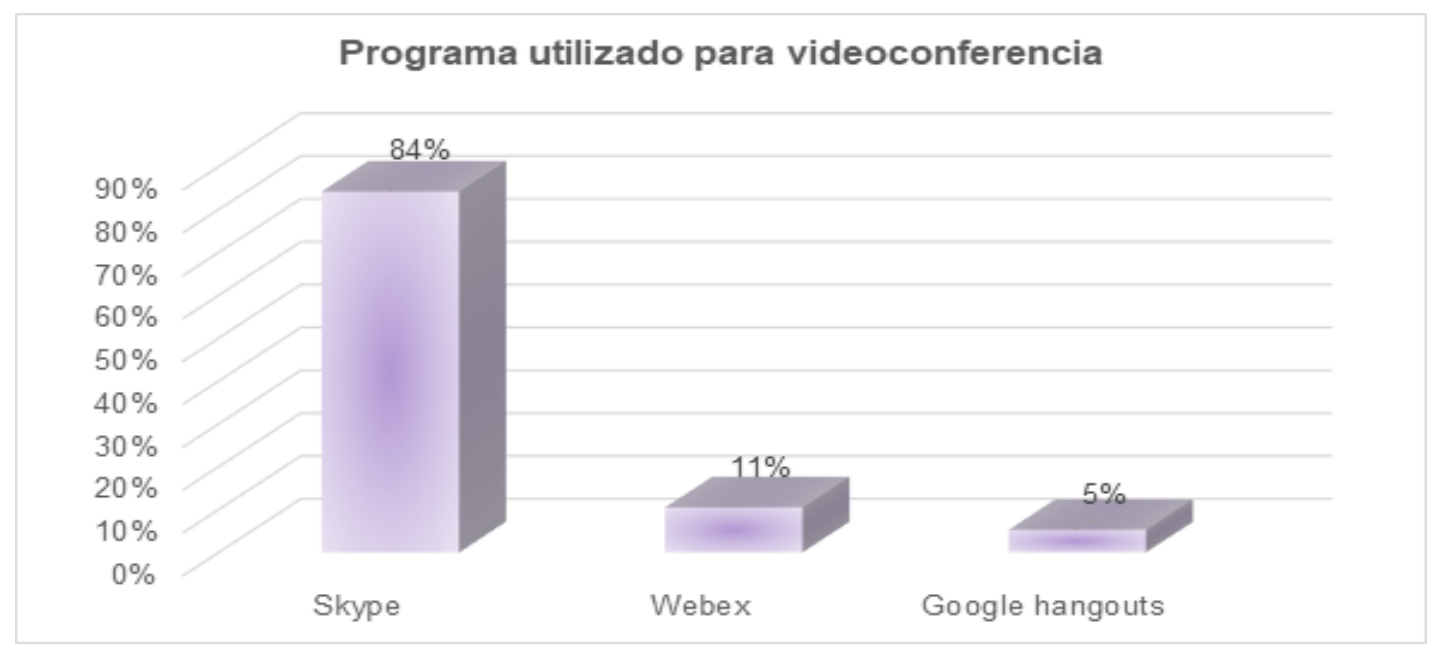

Fuente: Elaboración propia 

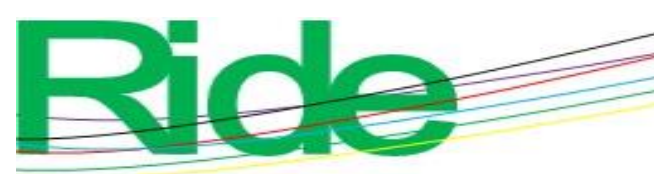

\section{Revista Iberoamericana para la Investigación y el Desarrollo Educativo ISSN $2007-7467$}

La figura 18 detalla si los estudiantes utilizan alguna plataforma educativa en la escuela: $53 \%$ lo hace regularmente, mientras que $15 \%$ solo eventualmente.

Figura 18. Frecuencia de uso de plataforma educativa en la escuela por los estudiantes

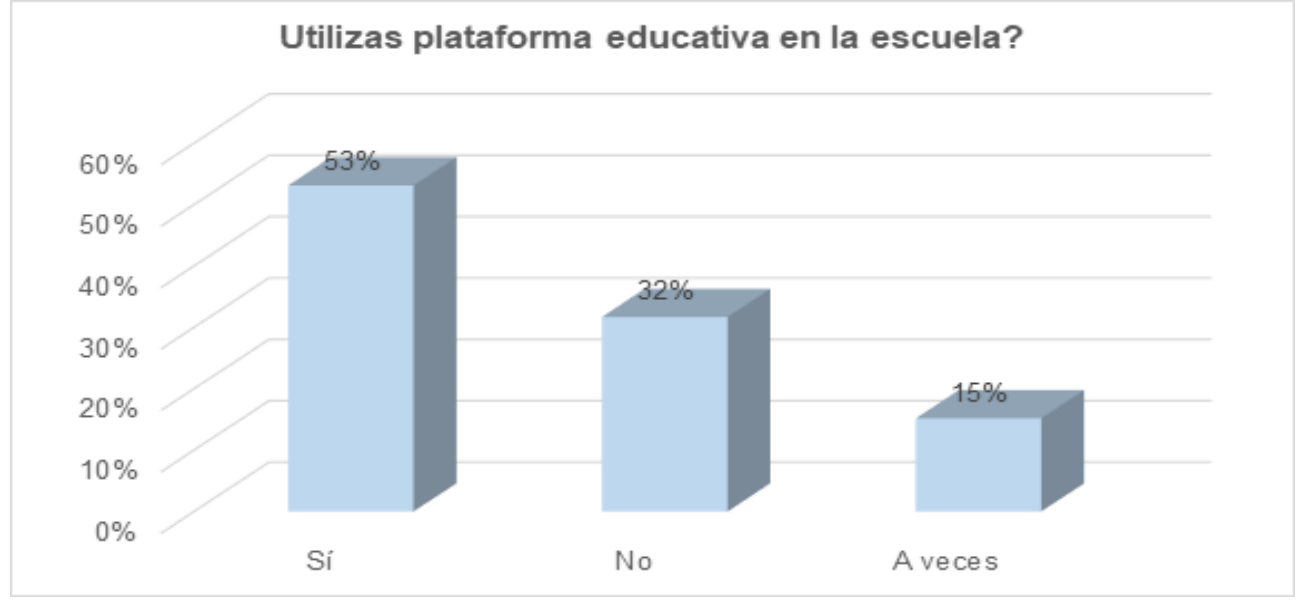

Fuente: Elaboración propia

La figura 19 muestra el tipo de plataforma educativa que utilizan los estudiantes. Campus virtual, que es la plataforma institucional, está por encima del resto. Sin embargo, es importante no dejar de mencionar que también utilizan alternativas como Canvas.

Figura 19. Plataformas educativas utilizadas en la escuela por los estudiantes

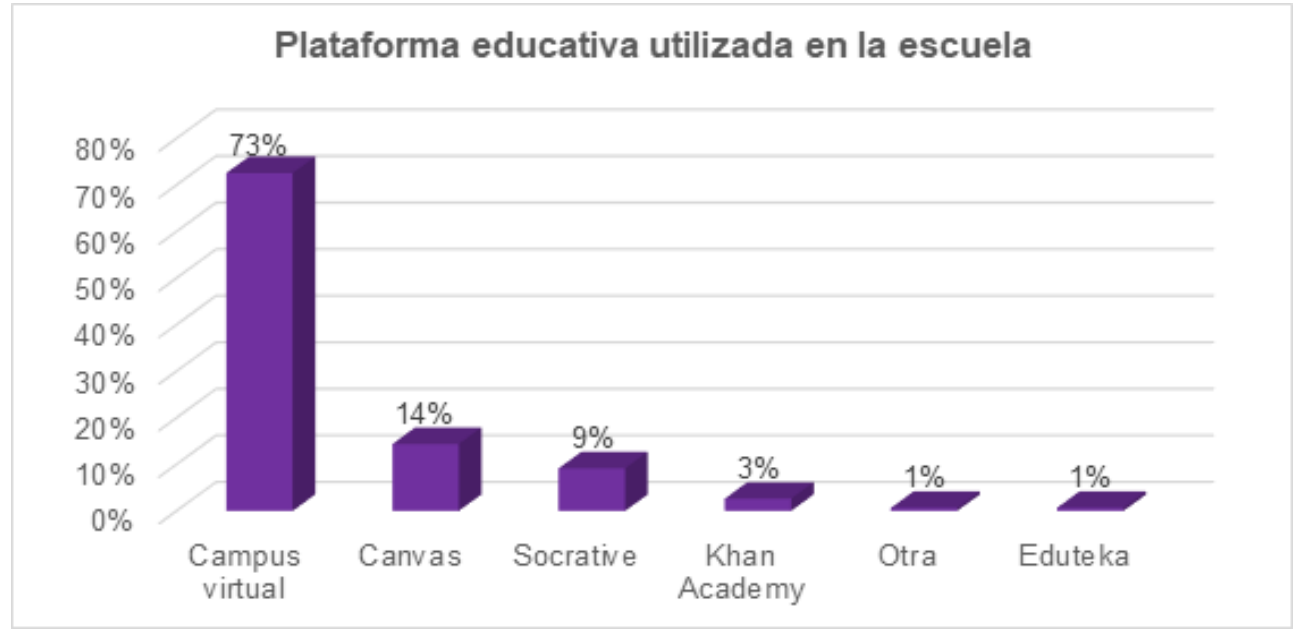

Fuente: Elaboración propia 

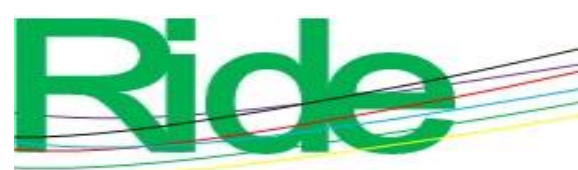

\section{Revista Iberoamericana para la Investigación y el Desarrollo Educativo ISSN $2007-7467$}

En la figura 20 se muestra el dispositivo que más utilizan los estudiantes en la escuela. La computadora y el teléfono celular se colocan en la punta.

Figura 20. Dispositivos utilizados en la escuela por los estudiantes

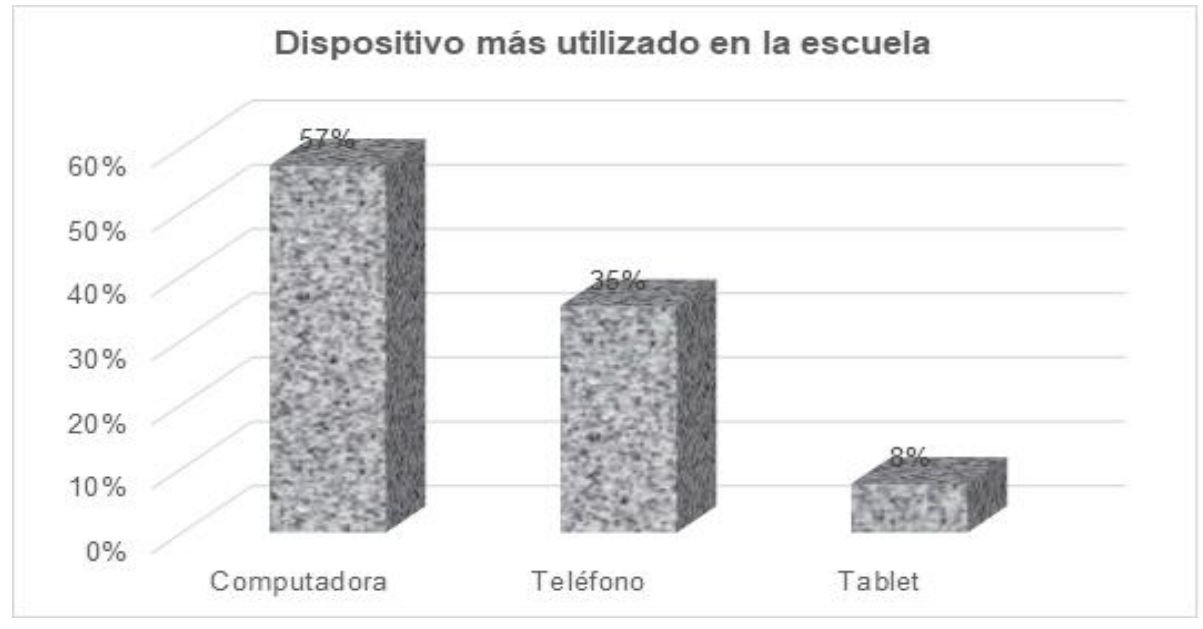

Fuente: Elaboración propia

La figura 21 muestra que las herramientas más utilizadas en general por los estudiantes en la escuela son Drive, Facebook y Youtube.

Figura 21. Herramientas utilizadas en general por los estudiantes en la escuela

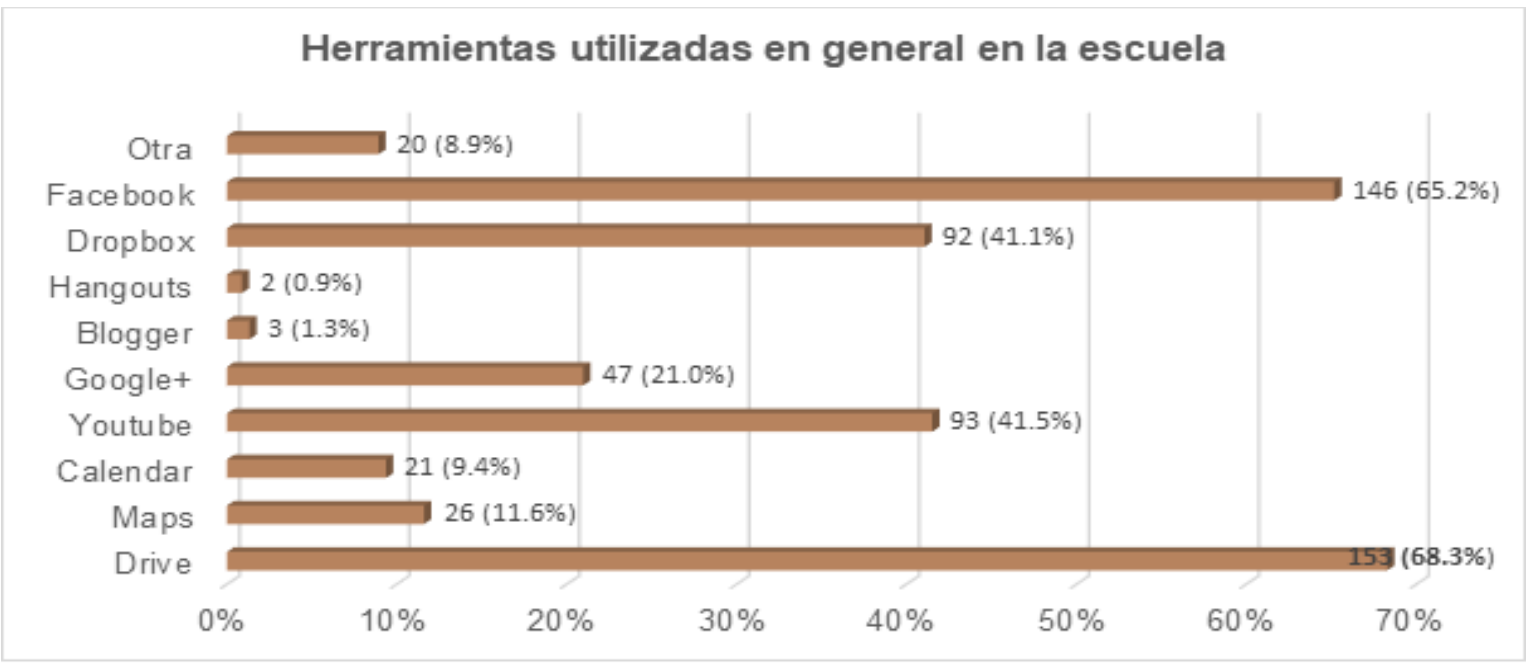

Fuente: Elaboración propia

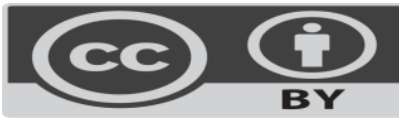



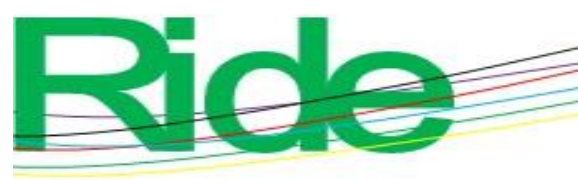

\section{Revista Iberoamericana para la Investigación y el Desarrollo Educativo ISSN $2007-7467$}

La figura 22 muestra las apps utilizadas en la escuela por los estudiantes: Whatsapp, Facebook y Youtube conforman el podio.

Figura 22. Apps más utilizadas por los estudiantes en la escuela

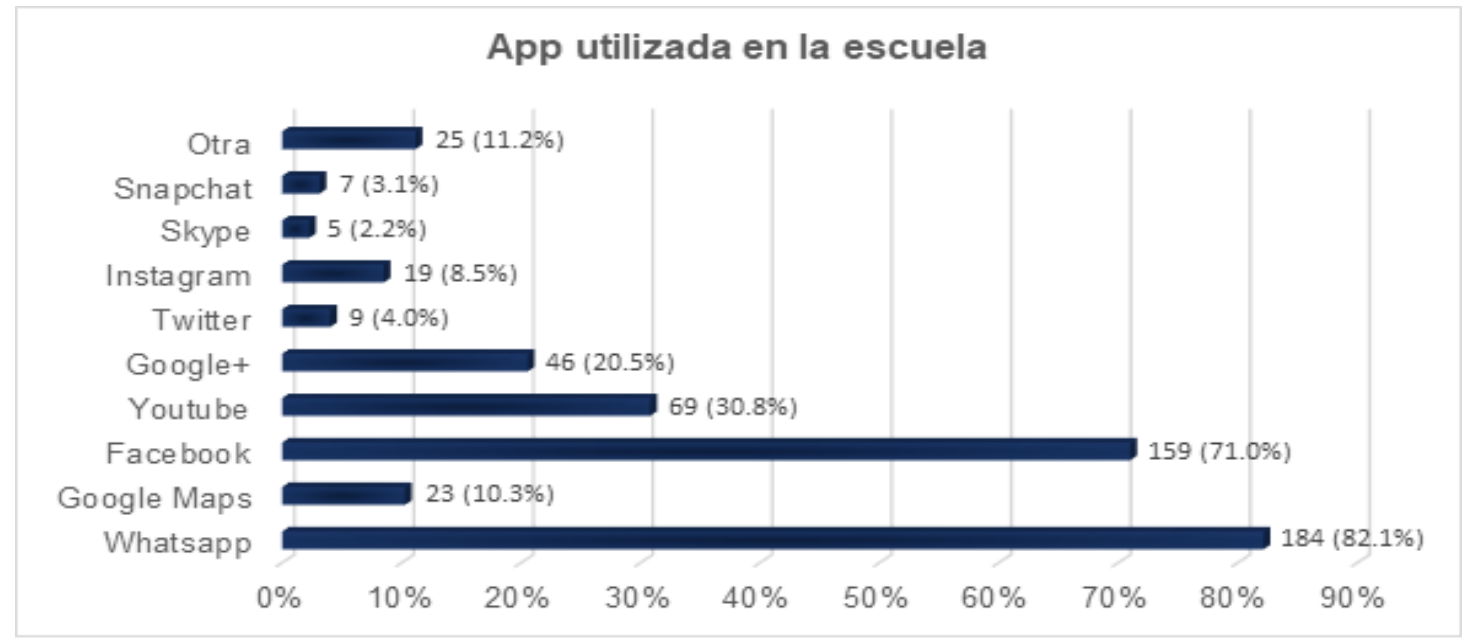

Fuente: Elaboración propia

En la figura 23 se muestran las herramientas que utilizan los estudiantes para crear cómics. Como se puede observar, las utilizan muy poco. A pesar de ello sobresalen ComicBook y Pixton.

Figura 23. Porcentaje de herramienta más utilizada por los estudiantes para crear cómics

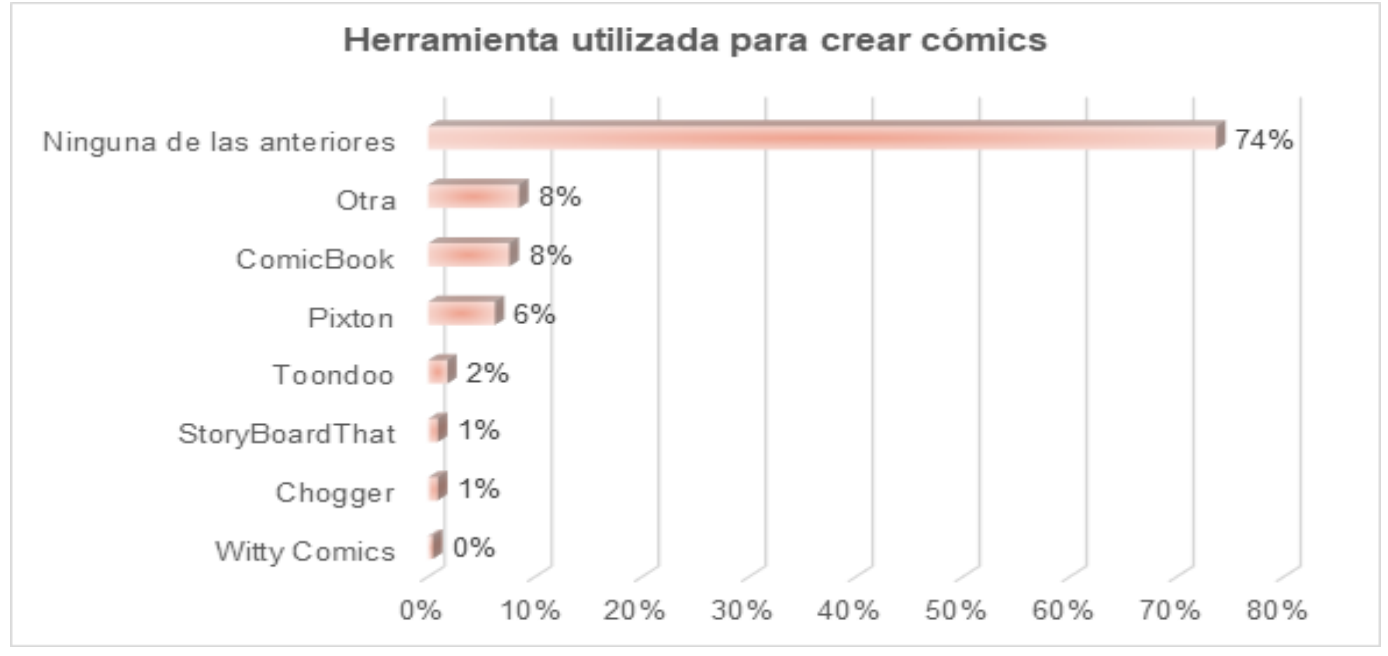

Fuente: Elaboración propia 

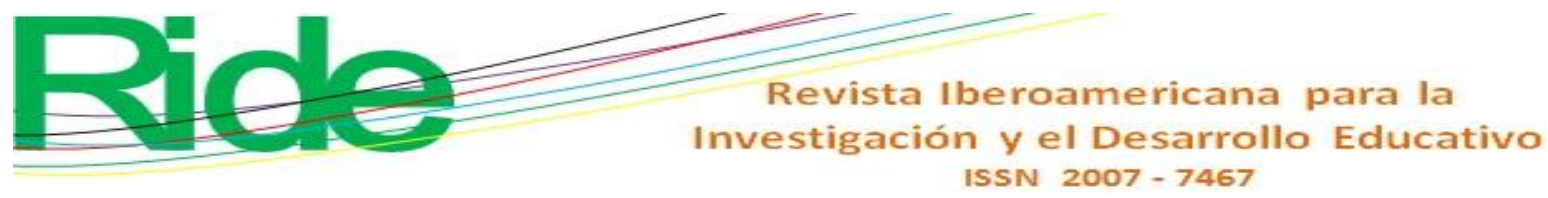

La figura 24 detalla el dispositivo que utilizan los estudiantes para algún juego. Sobresale el teléfono y le sigue la computadora.

Figura 24. Porcentaje de dispositivos más utilizados por los estudiantes para jugar

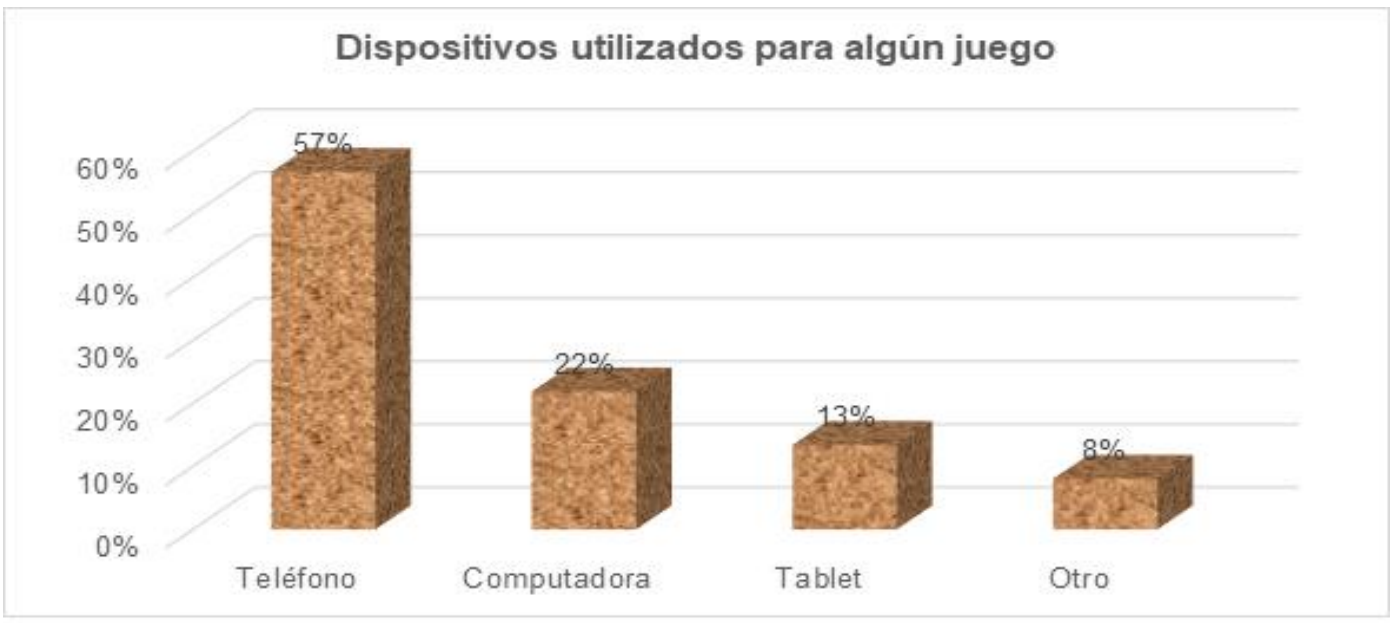

Fuente: Elaboración propia

Por último, la figura 25 muestra que $56.7 \%$ pasa el tiempo con un juego que no está especificado en las respuestas, pero en general todos juegan.

Figura 25. Juego utilizado por los estudiantes

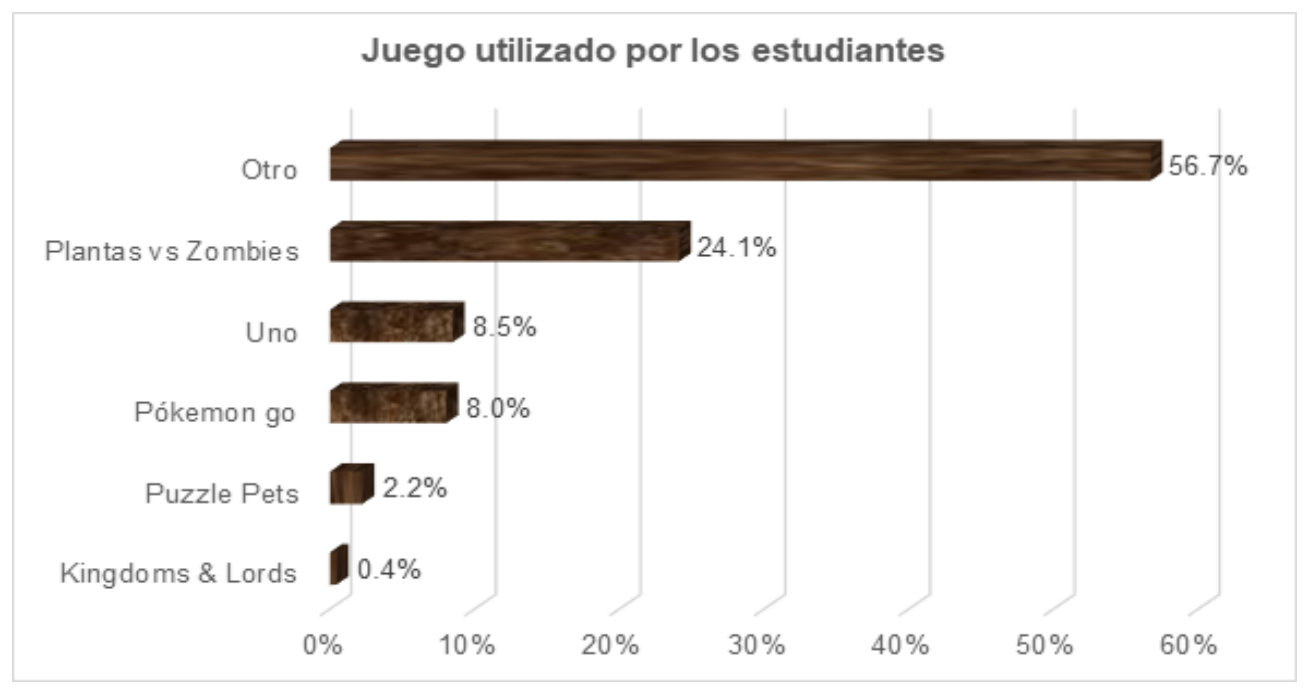

Fuente: Elaboración propia 

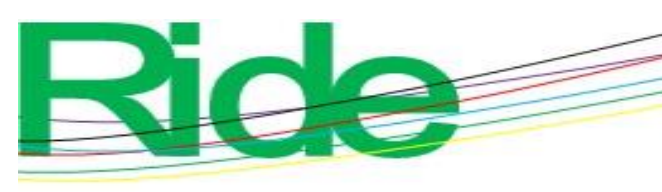

Revista Iberoamericana para la

Investigación y el Desarrollo Educativo ISSN $2007-7467$

\section{Discusión}

El presente trabajo de investigación tuvo la limitación de trabajar solo con una universidad pública, por lo que el proyecto está abierto a realizarse en otras universidades tanto públicas como privadas. Por otro lado, los datos obtenidos en esta investigación servirán para que los profesores estén informados de las herramientas utilizadas por los estudiantes y, una vez procesada esta información, puedan solicitar los trabajos en dichas herramientas y así apoyarlos en su proceso de enseñanza-aprendizaje, o bien como profesor comenzar a conocerlas, porque suele suceder que algunos profesores no están familiarizados con ciertas herramientas.

Es necesario tener en cuenta que se preguntó por las herramientas utilizadas en ese tiempo y momento, y que la tecnología va avanzando muy rápidamente; de hecho, cada vez más rápido surgen aplicaciones tanto para la computadora como para el teléfono, lo cual hace que los estudiantes vayan utilizando herramientas diferentes de un año a otro.

\section{Conclusiones}

Los estudiantes están relacionados con las herramientas tecnológicas, pues utilizan varias aplicaciones en la escuela y para su uso personal, así como dispositivos electrónicos como la computadora, teléfono y tableta.

El dispositivo que más utilizan los estudiantes en la escuela es la computadora, y las aplicaciones más usadas allí son Drive, Facebook y YouTube. En cuanto a las redes sociales, las más sobresalientes son Facebook, YouTube e Instagram. Las aplicaciones más utilizadas en el teléfono celular, mientras tanto, son WhatsApp, Facebook y YouTube.

Para las plataformas educativas, la más sobresaliente fue la institucional (Campus virtual); pero no solo utilizan esta, sino que también han llegado a utilizar otras como Canvas y Socrative. Y aunque la mayoría no han recurrido a las videoconferencias para sus clases, entre los estudiantes que sí la han utilizado sobresalió la herramienta Skype, seguida por Webex. En lo que se refiere a los navegadores de Internet más utilizados, el que sobresale es Google Chrome, con una gran ventaja sobre Mozilla y Safari.

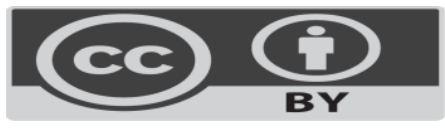


Cabe mencionar que, a pesar del tiempo que ha pasado, los programas de Microsoft Office siguen siendo los preferidos por los estudiantes, ya que el procesador de texto que más se utiliza es Word, seguido del procesador de texto en línea. Para los ejercicios estadísticos, el más utilizado es Excel, seguido por Minitab y el programa R. En lo que se refiere a las presentaciones, el que más sobresale es PowerPoint, seguido de Prezzi. Para los documentos en PDF, el programa que más sobresalió fue Adobe Acrobat y a continuación Nitro Pro.

Esto conduce a pensar que los estudiantes están muy involucrados con las herramientas tecnológicas, ya que, aunque sigan apareciendo nuevas aplicaciones, ellos estarán dispuestos a utilizarlas. Una de las aplicaciones más recientes es WhatsApp, y es de las que más utilizan. Los profesores tienen que involucrarse también con dichas herramientas y aplicaciones, puesto que los nuevos desarrollos posibilitan clases amenas y dinámicas. Es importante subrayar que las herramientas tecnológicas no solo les están brindando soporte académico, sino que incluso podríamos vislumbrar un soporte emocional y de entrenimiento. Este podría ser tema de investigaciones posteriores. Por lo pronto, la escuela puede ser una gran ganadora si observamos, planeamos y actuamos bien utilizando estratégicamente estas herramientas.

Con esto, los estudiantes pueden estar motivados durante su estancia en la universidad, además de que estarán involucrados en su formación al participar activamente en el proceso de enseñanza-aprendizaje, utilizando y aplicando herramientas para lograr un máximo aprovechamiento escolar, a la vez que aumentan su espacio social. Si el docente las utiliza y les ayuda a utilizarlas adecuadamente, el aprovechamiento podrá ser mejor. 


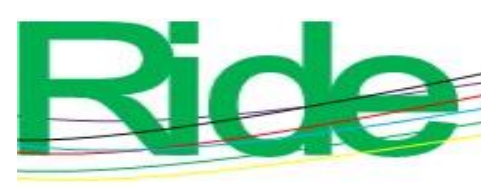

Revista Iberoamericana para la

Investigación y el Desarrollo Educativo

ISSN $2007-7467$

\section{Referencias}

Cabrol, M. y Severin, E. (2010). TICs en educación: una innovación disruptiva. Aportes, (2). Recuperado de https://publications.iadb.org/es/publicacion/14676/tics-en-educacion-unainnovacion-disruptiva.

Cassany, D. y Castellà, J. M. (2011). Aproximación a la literacidad crítica. Perspectiva, 28(2), 353374.

Cobo, C. y Moravec, J. W. (2011). Aprendizaje invisible. Hacia una nueva ecología de la educación. España: Publicacions i Edicions Universitat de Barcelona / Universidad Internacional de Andalucía.

Díaz, F. y Hernández, G. (2002). Estrategias docentes para un aprendizaje significativo (vol. 2). México: McGraw-Hill.

McMillan, J. H. y Schumacher, S. (2005). Investigación educativa: Una introducción conceptual. Madrid, España: Pearson educación.

Ministerio de Educación y Ciencia [MEC]. (2006). Real Decreto 1513/2006, de 7 de diciembre, por el que se establecen las enseñanzas mínimas de la Educación primaria. Recuperado de https://www.boe.es/boe/dias/2007/01/05/pdfs/A00677-00773.pdf.

Mishra, P. and Koehler, M. J. (2006). Technological Pedagogical Content Knowledge: A Framework for Teacher Knowledge. Teachers College Record, 108(6), 1017-1054. Retrieved from http://citeseerx.ist.psu.edu/viewdoc/download?doi=10.1.1.91.7 990\&rep=rep $1 \&$ type $=$ pdf.

Moravec, J. W. (2013). Knowmad society: The "new" work and education. On the Horizon, 21(2), 79-83. Retrieved from https://doi.org/10.1108/10748121311322978.

Moreira, M. A., Salvat, B. G. y García, M. Á. M. (2008). Alfabetizaciones y tecnologías de la información y la comunicación. Madrid, España: Síntesis.

Necuzzi, C. (2013). Estado del arte sobre el desarrollo cognitivo involucrado en los procesos de aprendizaje y enseñanza con integración de las TIC (vol. 8). Argentina: Fondo de las Naciones Unidas para la Infancia (Unicef). 

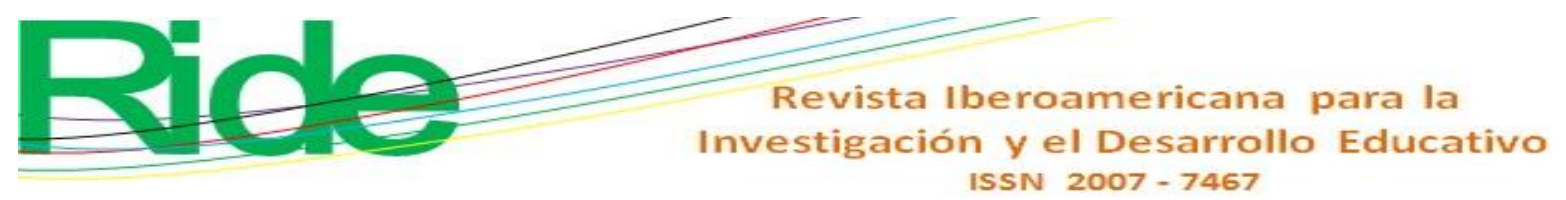

Organización de las Naciones Unidas para la Educación, la Ciencia y la Cultura [Unesco]. (2008). ICT Competency Standards for Teachers. Organización de las Naciones Unidas para la Educación, la Ciencia y la Cultura.

Pico, S. (2013). Formación TIC del profesorado para garantizar el éxito en la integración de la tecnología. Ítaca. Revista de Filologia, 4, 65-80.

Sangrá, A. y González, M. (coords.) (2004). La transformación de las universidades a través de las TIC: discursos y prácticas. Barcelona, España: Editorial UOC.

Siemens, G. (2014). Connectivism: A learning theory for the digital age. International Journal of Instructional Technology \& Distance Learning.

Tejedor, F. J. T., y Muñoz, A. G. V. (2006). Competencias de los profesores para el uso de las TIC en la enseñanza. Análisis de sus conocimientos y actitudes. Revista española de pedagogía, 64(233), 21-43. 


\begin{tabular}{|c|c|}
\hline Rol de Contribución & Autor (es) \\
\hline Conceptualización & María del Carmen Molinero Bárcenas. \\
\hline Metodología & María del Carmen Molinero Bárcenas. \\
\hline Software & N/A \\
\hline Validación & María del Carmen Molinero Bárcenas \\
\hline Análisis Formal & María del Carmen Molinero Bárcenas. \\
\hline Investigación & María del Carmen Molinero Bárcenas. \\
\hline Recursos & María del Carmen Molinero Bárcenas (Principal) / Ubaldo Chávez Morales (Apoya) \\
\hline Curación de datos & María del Carmen Molinero Bárcenas. \\
\hline $\begin{array}{l}\text { Escritura - Preparación del borrador } \\
\text { original }\end{array}$ & María del Carmen Molinero Bárcenas. \\
\hline Escritura - Revisión y edición & María del Carmen Molinero Bárcenas (Igual)/ Ubaldo Chávez Morales (Igual) \\
\hline Visualización & María del Carmen Molinero Bárcenas. \\
\hline Supervisión & Dr. Ubaldo Chávez Morales \\
\hline Administración de Proyectos & María del Carmen Molinero Bárcenas (Igual)/ Ubaldo Chávez Morales (Igual) \\
\hline Adquisición de fondos & María del Carmen Molinero Bárcenas (Principal) / Ubaldo Chávez Morales (Apoya) \\
\hline
\end{tabular}

